\title{
Theory, Experiment and the Federal Communications Commission Spectrum Auctions
}

\author{
Jeffrey Banks*, Mark Olson**, David Porter**, Stephen Rassenti** and Vernon Smith** \\ * Division of Humanities and Social Science 228-77, California Institute of Technology, \\ Pasadena, CA 91125, USA \\ ** Interdisciplinary Center for Economic Science, George Mason University, 4001 Truland \\ Building, 3330 Washington Boulevard, Arlington, VA 22201,USA
}

\begin{abstract}
The Federal Communications Commission uses an ascending bid auction called the Simultaneous Multi-round Auction (SMA) to assign spectrum for personal communication service licenses. Congress recently mandated that the SMA be evaluated to determine if it could be modified to allow "combinatorial" bids for packages of licenses. We review the theoretical background and prior experimental evidence relevant to the SMA procedures and their inherent defects which are driven largely by the presumption that values are common or affiliated and that bidder identities must be revealed in real time. We present results from experiments to evaluate the SMA and some its more important rules, along with a comparative test of the SMA with a combinatorial auction specifically designed for the Federal Communications Commission by Charles River and Associates. We find that several of the SMA rules hinder efficiency and create a trade-off between efficiency and time to complete the auction. In addition, when license values are superadditive the combinatorial auction outperforms the SMA, but requires much more time to complete and is not robust with respect to boundary cases.
\end{abstract}

JEL classification:

Keywords:

Please send proofs to:

Dr. David Porter

Interdisciplinary Center for Economic Science

George Mason University

4001 Truland Building

3330 Washington Boulevard

Arlington, VA 22201 
The US Congress has mandated an independent evaluation of the Simultaneous Multiround Auction (SMA) used by the Federal Communications Commission (FCC) to award spectrum licenses to bidders. This evaluation was required to include an experimental study that would increase understanding of the problems and the complexities of the SMA and examine alternatives to the SMA that might better facilitate the acquisition of efficient combinations of the elementary licenses where complimentarity is important. Part 1 provides a brief review of several issues, and some previous experimental findings that bear most directly on the conceptual and behavioral foundation of the FCC auction design problem. Part 2 contains results from experiments that examine certain features of the SMA auction. Part 3 provides an experimental examination of a combinatorial auction designed by Charles River Associates for the FCC as part of its mandate to examine auctions for licenses that have complimentary values.

\section{Part 1: Previous Experiments and Conceptual Issues Relevant to Spectrum Auctions}

\subsection{Economics of English (Progressive) Auctions}

We begin with a general review of the theory of English ${ }^{1}$ progressive auctions, under alternative assumptions about auction values: (A) individuals' valuations for an auctioned item are private and independent, or (B) individuals' valuations are common (or affiliated).

\subsubsection{Independent Private Values}

In this environment there are both advantages and disadvantages to the English progressive auction in comparison with sealed-bid (first or second price) or Dutch auctions. The principal advantage of the progressive auction is the transparency of the optimal bidding strategy. The auction requires minimal bidder sophistication for choosing a non-collusive strategy, and bidders have no incentive to invest in acquiring information as to other bidders' values or strategies. As a bidder who has estimated your maximum willingness-to-pay value for an item, you merely follow two simple (dominant strategy) rules: (i) after the first bid has been announced, and at all times until the auction ends, if the standing bid is below your value, you should raise your bid by the minimum bid increment as long as your new bid is not greater than your value; (ii) never raise your own standing high bid. This transparency and strategic simplicity account for the fact that the vast majority of auctions are of the English form both on 
and off the Internet (Cassady, 1967; Lucking-Reiley, 1999). The implications of (i) and (ii) guarantee theoretically that the bidding on each item auctioned will stop as soon as the bidder with the highest private value ( $\left.v_{1}\right)$ bids $b^{*} \geq v_{2}$ (the second highest value), given that the minimum bid increment is sufficiently small. No one will rationally invest in learning about the values or bidding strategies of other bidders because these will be revealed costlessly by the open bidding procedure. We note that the two-part strategy is not quite the same as stating that a bidder's dominant strategy is to bid actively until the price reaches the value of the object to him. The latter is correct for all but the highest value bidder, who, following rule (ii), discovers that he need bid only high enough to displace the second highest valuation bidder. When bidders do not announce bids from the floor as in the English clock auction (which historically seems never to have been successfully adopted in the field (Cassady, p. 196)) the clock ticks up until all but one bidder drops out; then stops at the winning price. As discussed below, for over a decade experimentalists have regularly used this procedure when they want to implement an auction procedure that will induce participants to reveal their true willingness-to-pay.

There are, however, prominent disadvantages of the progressive auction: (i) if bidders are risk averse then revenue will be higher in the first price sealed bid auction; (ii) the procedure has high transactions' cost in the sense that bidders must be present at the auction, tending to it in real time ${ }^{2}$ and (iii) it greatly facilitates the opportunity for collusive arrangements, or "rings" among the bidders, a fact that accounts for much of the colorful history of auctions. (See Cassady, pp. 177-192 on rings, and pp. 212-218 for other forms of collusion routinely observed in the history of English auctions). In addition, Porter and Vragov (2000) show that demand reduction (the strategy of under-revealing demand for multiple units of a good) is much more prevalent in the English auction than sealed bid auctions).

Thus, the great transparency of the English auction, if you know who is bidding, and how much, enables the formation of collusive arrangements in real time, reducing uncertainty about who to communicate with in advance of the auction. The English auction provides free real-time information that enables ring members to identify those who are not living up to their collusive agreement. It also allows ring members to determine who among their competitors outside the ring should be included in their ring at subsequent auctions. The English auction also enables collusion at auction time in the complete absence of prior agreements or communication: associates who know each other find it natural not to raise each other's bid. Parallel 
considerations apply to sealed-bid auctions where the seller announces all bids after the auction. Thus, bidding rings can readily determine who is or is not complying with the agreement, and who are the outsiders that have won so that they can be included in subsequent rings. Outer continental shelf petroleum lease bids are publicized after each auction by the Government, thereby providing such costless information service to any ring. (Cox, Isaac and Smith, 1983).

The essence of the English auction is that the successive bids are known to all. There is no need for the bidder to be publicly identified. For this reason auction houses permit bidders, by pre-arrangement with the auctioneer, to use signals to transmit bids. Such practices are widespread among auction houses and have two important consequences: (1) they make collusion more difficult, and (2) if bidder valuations are not independent, secrecy in bidding allows asymmetric information to remain private. By bidding covertly bidders who are known or believed by others to be better informed are protected from revealing any asymmetric information. The effect of (2) is to lower the selling price, while (1) raises it. These observations are relevant to the various FCC auction mechanisms, as they have evolved, since the original designers believed asymmetric information to be potentially so important that bidder identities were made public to reinforce common values. This facilitated gaming (strategic manipulation) and collusion. The subsequent attempt to control such behavior has led to more complex rules and constraints on bidding, which induce new forms of gaming, and attempts to control these with new rules, and so on. (See Milgrom, 2000 and McAfee and McMillan, 1996).

All of the above considerations apply to offers of multiple units in English auctions under either of the two simultaneous auction procedures suggested by Vickrey $(1961,1976)$. The case in which multiple units are offered, discussed below, has led quite prominently to the phenomenon of "jump bidding," originally observed in laboratory experiments by McCabe, Rassenti and Smith (1988). This prompted them to study English Clock auctions for multiple unit auctions (where each bidder demands one unit).

\subsubsection{Common Value Auctions}

In a widely cited path breaking theoretical contribution, Milgrom and Weber (1982) introduced the concept of the common value auction, as distinct from the independent private values auction. The paradigm here is the "mineral rights" problem: bidders for rights to explore for oil, gas and minerals on land or off-shore tracts, each estimate (e.g. by seismic readings in the 
oil and gas industry) the quantity of the recoverable mineral, if any, and base their bids on their own estimates. Thus, Milgrom and Weber (1982, p. 1093) assume that "To a first approximation, the values of these mineral rights to the various bidders can be regarded as equal, but bidders may have differing estimates of the common value."

Suppose the value of an auctioned item to individual $i$ is of the form

$$
\widetilde{V}_{i}=\alpha \widetilde{P}_{i}+(1-\alpha) \widetilde{v}
$$

where $\widetilde{P}_{i}$ is the item's uncertain, strictly private, value to $i$, and $\widetilde{v}$ is the item's uncertain common value to all bidders. The distribution of $\widetilde{v}=\widetilde{v}\left(s_{1}, \ldots, s_{N}\right)$ is assumed to depend upon information (signals), $s_{i}$, available to each $i$. As indicated, Milgrom and Weber (1982) assume as a first approximation, that $\alpha$, a characteristic of the commodity, is approximately zero. Although this is a useful abstract exercise in developing the theory of auctions, and providing an understanding of possible issues in auction design, we are lacking in objective, testable criteria for determining $\alpha$. There are no systematic procedures for identifying $\alpha$ from the observed characteristics of an industry. That $\alpha$ is indeed greater than zero in applications to mineral extraction industries is supported by the observation that oil and mineral companies buy and sell proven reserves. Thus an oil company whose marketing proficiency outstrips its supply capability may value a petroleum reserve more highly than the selling company. Indeed, empirically it may be that $\alpha$ is closer to unity, than to zero in the petroleum sector.

For mixed value environments of the form (1) when $0<\alpha<1$, we have decisively negative theoretical results: a bidding equilibrium does not exist in auctions defined in this mixed environment. The difficulties are foundational. "With multidimensional types (as represented by $\widetilde{P}_{i}$ and $\widetilde{v}$ in (1)), inference from prices may be non-monotone and complicated. It may not be possible to order the bids of agents with multidimensional information and incentives in a way that is consistent with what they can infer from prices and their resulting incentives." (Jackson, 1999, p. 8; also see Klemperer, 1998). An important implication of this is that it cannot be claimed that (although we have no equilibrium theory for the FCC auctions) game theoretic "intuition," developed from complete information examples, allows valuable guidelines to be articulated about auction design (McAfee and McMillan, pp. 172-3). Another implication is that confidence in the postulated advantages of the open English format is undermined. 
The principal theoretical result that follows from the hypothesis that individual valuations are common can be stated as follows: prices are higher in the English auction than in the first price (or Dutch auction, which is strategically equivalent to the first price) and the second price sealed bid auctions. Prices are higher because each bidder's private information on the common value is valuable information to other bidders, and revealed in the open bidding process. This potential pooling of private information to produce a higher revenue outcome is the essential feature that drives the judgement that English auction procedures dominate the alternatives. That judgement, however, is based on the assumption that $\alpha$ is near zero, and formal theory alone cannot say why this should be so.

We note several possible or potential contraindications that provide a basis for doubt concerning the apparent superiority of the English auction conditional on values being common.

1. If information not already publicly known is costly to acquire, and all bidders are both rational and sophisticated, then bidders are not well-motivated to invest in acquiring information if they will subsequently be compelled to reveal it in an open bid. It appears that all but the high bidder, given that the investment is a sunk cost, will surely reveal the value implications of their information. If so, then the decision problem devolves to assessing the probability of being the high bidder, then computing how much investment in information is justified by weighing cost against that expected benefit. Sophisticated bidders, using consulting firms and economic theorists, and applying the principle of backward induction, will anticipate this future state of the world and refrain from investing in the acquisition of information that is not private to their own special circumstances. We lack a theorem that addresses these needed extensions of the theory.

To the extent that the many sophisticated bidders in the FCC auctions expended resources for acquiring information on technology and demand, we are led to deduce that this was a consequence of the judgement that the information enhanced the private value of the licenses to the individual bidder.

2. This line of reasoning suggests that the common value information that will be revealed in the bids will be that which is dispersed initially among the bidders as a consequence of technological, institutional and legal activities that had been undertaken for other business purposes, and are then discovered to be complimentary to the commodity (or right) that is proposed for auction. But investment in such activities is justified independently and prior to the sale of the commodity. 
3. Ironically, it follows that buyer rings or consortia are not prima facie to be ruled out in common value auctions with costly information. Such consortia, if they pool the information of their members, provide an incentive to invest in information acquisition by reducing the freerider problem. Rings can improve efficiency if there are subsets of the bidders whose commonality is distinct from that of others. To the extent that a common private value $\tilde{v}_{K}$ for each bidder of subset $K$ is a technological or marketing reality then allocations can be improved by allowing consortia to each internalize these effects through joint ventures that then bid competitively against each other. Optimality is restored by competition among the consortia that have alternative private uses for the resource.

4. Given the hypothesis that commodity ' $\mathrm{X}$ ' has common value components, one can evaluate alternative auction procedures, deriving theorems, as in Milgrom and Weber, showing the implication of common valuedness for auction performance. What the theory does not tell you is the answer to the following: given commodity ' $\mathrm{X}$ ' and a description of its operating and technological characteristics, what is the value of $\alpha, 0 \leq \alpha \leq 1$, in equation (1)? In particular, is $\alpha$ small enough to justify the choice of English over other auction forms especially given the implications above for investment in information acquisition? Clearly there must be a trade-off here. The fact that the English auction provides the greatest scope for collusion among sophisticated bidders, yielding lower prices and efficiency, and requires high transactions' (monitoring/participation) cost, must be weighed against the effect of the postulated size of $\alpha$. Is $\alpha$ low enough for the revenue advantages of the English auction, under common valuedness, to outweigh its disadvantages?

5. In auction theory where the value of the auctioned item to oneself or others is uncertain, this uncertainty is resolved at the end of the auction. This is the basis on which experimental studies have tested hypotheses derived from common value auction theory; i.e., we can create these postulated conditions in the lab. But suppose, after the sale of commodity ' $X$ ' at auction, the main uncertainty that is resolved is who won and who lost the rights awarded, with the winners still facing most of the uncertainty as to the value (common or private) of such rights. How should this reality affect the choice of auction format? Clearly, if most of the uncertainty will be resolved subsequently, based on "signals" subsequently purchased, this ex post auction information cannot be revealed for pooling purposes during the auction and the case for English procedures is further compromised. 
6. We note, finally, that in a common value auction environment efficiency is irrelevant -any allocation to any firm is efficient - the issue is exclusively one of maximizing revenue by incentivizing the public revelation of all information. There is a direct contradiction between the stated primary function of the FCC spectrum auctions - efficiency - and the FCC's interpretation, argued above, that common valuedness is the predominating characteristic of the commodity. Hence, when common value elements are important, alternatives to the English procedure are more likely to impair revenue, not efficiency.

\subsection{Review of Relevant Experimental Results}

This section provides a short summary of those well-established experimental findings that are directly relevant to the spectrum auctions. These findings cover: (i) behavior in standard single object auctions; (ii) behavior in common value auctions; (iii) a winner's curse in private value English auctions for gambles; (iv) jump bidding, impatience, inefficiency and manipulation attempts in claimed "incentive compatible" multiple unit English auctions; and (v) combinatorial auctions.

\subsubsection{Standard Single Object Auctions}

Behavior in experimental private value English, Dutch, first and second price sealed bid auctions was originally reported in Coppinger, Smith and Titus (1980; reprinted in Smith, 1991; also see Kagel and Roth, 1995, Chapter 7 for a comprehensive survey). English auction prices and efficiencies were found to vary insignificantly from the predictions of theory; i.e., most awards were to the highest value bidder at prices reasonably near to the second highest value. A very few awards, somewhat above the second highest value, could be attributable to minor "jump bidding" in the sense that bidders, free to select the amount by which they raised the standing bid, sometimes chose an increment larger than necessary. (Jump bidding is much more serious in multiple unit English auctions as discussed below). A few awards were at prices well below the second highest valuation (Coppinger, Smith and Titus, Chart 2). These were believed due to subjects who knew each other and refrained from raising each other's bid, which was verified in one case. First price sealed bid auction prices were significantly higher than those for the second price sealed bid auction. 
A more extensive comparison of 780 Dutch, first and second price sealed bid auctions in an environment in which values are rectangularly distributed is reported by Cox, Roberson and Smith (1982; reprinted in Smith, 1991). Again, first price auctions yield higher prices than second. Also Dutch prices are found to be significantly lower than those in first price auctions. Efficiency was highest in second price auctions (94\% of the awards were Pareto optimal), lower in first price auctions (88\%) and lowest in Dutch auctions (80\%).

\subsubsection{Common Value Auction Behavior}

Initial experimental studies of first price sealed-bid auctions with common values (Kagel and Levin, 1986) identified serious problems with subjects not discounting properly their private information, except in small groups of 3-4 bidders, and suffering the "winner's curse." All bidders receive unbiased estimates of the value of the object, but the highest of several estimates is biased upward. This "order statistic" property requires that the optimal bid take this property into account. Hence, the Nash equilibrium bid of each bidder is below each bidder's private estimate. Inexperienced subjects tend to bid their signals' indicated value or more. Consequently, the highest signal bidder wins, but pays too much and on average loses money.

However, this preliminary finding in Kagel and Levin did not hold up with experienced bidders. Cox and Smith (1992) found that experienced bidders (who could elect to enter the auction, or eliminate themselves and receive a modest return each period) tended to bid below their estimates of value, and make positive profits. Subsequently, Garvin and Kagel (1994) confirmed these results in their study of "learning" (experience) in common value auctions. It is now well established that in most cases even unsophisticated bidders with training and experience have no significant difficulty overcoming the winner's curse.

Kagel and Levin (see Kagel and Roth, p. 548) report common value auction results comparing first price with English auctions. Average prices in the English auctions were lower than in the first price sealed bid auctions, and both were higher than the expected common value, yielding negative profits under both auction procedures. But losses were lower in the English auction. Consequently, the winner's curse was reduced but not eliminated in English auctions. It was not shown whether it would be eliminated by using more experienced subjects who could choose between bidding in the auction or engaging in an alternative return activity. 


\subsubsection{A "Winner's Curse" in Private Value English Auctions for Gambles}

Two studies (Chew and Nishimura, 1999; Berg, Dickhaut and McCabe, 1996) have reported results from English auctions of objects of uncertain value - gambles yielding alternative monetary prizes with known stated probabilities. The two sets of results are qualitatively very similar although the auction formats, and commodity gambles are quite different. Also, Cox and Grether (1996), incidental to their study of preferences reversals, report similar findings. All three report English auction prices in excess of the expected value of the auctioned gamble. This supports the conclusion that uncertainty in private value auctions leads to overbidding and losses.

\subsection{4 “Jump Bidding” and other Behavioral Problems in Multiple Unit English Auctions}

"Jump bidding" in the form of bid increases higher than the minimum required increment, and raising your own standing high bid ("upping yourself”), occurred in all of the first three FCC auctions. It occurred most notably in the first two narrowband license offerings, but less in the third (wideband) auction. McAfee and McMillan (pp. 168-9) suggest that this might have been the result of "learning" or the higher value of the widebands. We think it just as likely due to the scarcity of bidders. Though standard theory views such bidding behavior as transparently irrational, it has been observed as early as 1988 in multiple unit experimental auctions.

Vickrey (1961) proposed an extension of the single object English auction for the multiple unit case (each bidder desiring at most one of the $m$ homogeneous units offered for sale) as follows:

In simultaneous auctioning the $m$ items can be put up simultaneously, and each bidder permitted to raise his bid even when this does not make his bid the highest. When a point is reached such that no bidder wishes to raise his bid further the items are awarded to the $m$ highest bids. . . . Bidders with the top $m$ values then secure the article at a uniform price equal to the $(m+1)$ st value; the result is again Pareto-optimal.

We note that this mechanism does not require or impose a uniform price. When $m=$ 1 in the single object English auction, the highest value bidder, never raising the price by more than the minimum increment, will not need to bid higher, once her bid equals or just exceeds the second highest value. Similarly, in the above quotation, Vickrey is stating 
that the $m$ highest value bidders will each raise their bid only to the level of the $(m+1)$ st highest value (or just above), and as a consequence, they will each be awarded an item at the common bid price equal to the $(m+1)$ st highest value.

In their comparison of eight variations on English multiple unit auctions, McCabe, Rassenti and Smith $\left(1988,1990\right.$; hereafter MRS) ${ }^{3}$ refer to this version of the Vickrey procedure as the Simultaneous Bid Auction, which is similar to the Japanese hand signal auction for selling fish in single lots, and the Japanese electronic auctions for fruit and vegetables (Cassady, pp. 63-66; 197-198).

Subsequently, Vickrey (1976) modified his earlier proposed simultaneous auction for $n$ units (referred to as the Vickrey Matching Auction by MRS p. 52) as follows:

A Pareto optimal procedure is available, however, if all the items are auctioned simultaneously, with up to $n$ bids permitted at any given level, the rule being that once $n$ bids have been made equal to the highest bid, any further bid must be higher than this. Within the 'jitter' determined by the minimum acceptable bid increment, this assures optimal results, ...

The results are optimal because once $n$ matching bids reach the $n+1^{\text {st }}$ value, no one will raise the bid. In all of the experiments reported by MRS 4 units are offered for sale to 10 bidders whose private resale values are drawn independently with replacement from a uniform distribution with support $[1,224]$. (See MRS, Table 1, p. 46). These facts are common information to all subjects.

When MRS ran the "Pareto optimal" Vickrey Matching Auction (and the Simultaneous Bid Auction above) they "found that prices were higher than predicted, and in general, subjects were raising their bids by too large an amount. When one of the three highest value subjects raises the suggested price $\mathrm{p}^{\mathrm{t}}$ by too much, all four units may not trade." (MRS, p. 54) Their results, showing the effect of such jump bidding, under several treatment variations on Vickrey's proposals, are displayed in a series of figures along with those for the English Clock auction which emerges as uniformly the best procedure studied by MRS (see their figures 1, 2, 4-8).

Finally, MRS implemented a backtracking procedure (Vickrey Backtracking), allowing the price to fall, whenever there was an overshoot with less than four bidders in the contract. This "fix" greatly improved allocations, but there was gaming by some subjects who anticipated that the price would overshoot then fall, and less-cautiously jumped the bid by too much. The 
major problem with the procedure was that it took a much longer time relative to the other auctions, as backtracking was widely employed, and transactions' cost escalated. These experiments illustrate the potential to initiate the following dangerous design cycle. You begin with a nice, theoretically "optimal" auction procedure. In implementation, you encounter behavioral incentives or "strategic" problems not considered as part of the original theory and likely intractable from a theoretical point of view. You come up with a rule "fix," to provide a countervailing incentive. You create a new problem requiring a new rule adjustment, and so on, in a process that need not converge.

In the case above, all problems arose from a common feature: bidder control over bids from the floor. This issue, not readily anticipated by formal analysis, surfaced naturally in experiments, and made ex post sense. The bottom line, transparently evident in the MRS figures, is that if you want to do English multiple-unit (incentive compatible) auctions the way to do them is to use the English Clock. In 44 English Clock auctions only one failed to award the item to the highest value buyers. This method dominates all others in terms of efficiency (percent of maximum possible joint payoff that is realized by the participants). There can be no jump bidding because no one can bid a price. Thus, MRS (p. 70) conclude "that the English Clock is our best implementation and is likely to find acceptance in the field. This auction gives participants feedback during the auction, ..., produces consistent pricing and very high efficiency, (and) can accommodate programmed (or electronic) . . . bidding”. Essentially, the procedure works well because it removes from bidders the right to announce bids from the floor - they can only indicate willingness to be in, or out, at the standing price, and once out they cannot reenter (in the MRS implementation). Bidding from the floor invites jump bidding, collusion and longer auctions. Jump bidding is encouraged by impatient bidders who may sacrifice potential profit in their desire to speed up the pace of the auction and reduce their transactions' cost (MRS, p. 70-72, offers a model of impatience). Some bidders may jump bid as a bluff: "I intend to win so you might as well give up," but this is entirely conjectural. Such "signals," however, are not rational, and we think that they are not a plausible comprehensive explanation of jump bidding. Among sophisticated bidders where it is seen as not too costly to jump, we would conjecture that it is a way of distracting the attention of other bidders and causing speculation about why they did it. 


\subsubsection{Combinatorial Auctions}

The general concept of smart computer-assisted markets, and the specific version known as a "combinatorial auction," originated in the early 1980s. (Rassenti, 1981; Rassenti, Smith and Bulfin, 1982; hereafter RSB). As noted by RSB (p. 672):

To our knowledge, this study constitutes the first attempt to design a "smart" computer-assisted exchange institution. In all the computer-assisted markets known to us in the field, as well as those studied in laboratory experiments, the computer passively records bids and contracts and routinely enforces the trading rules of the institution. ${ }^{4}$ The RSB mechanism has potential application to any market in which commodities are composed of combinations of elemental items (or characteristics). The distinguishing feature of our combinatorial auction is that it allows consumers to define the commodity by means of the bids tendered for alternative packages of elemental items. It eliminates the necessity for producers to anticipate, perhaps at substantial risk and cost, the commodity packages valued most highly in the market. Provided that bids are demand revealing, and that income effects can be ignored, the mechanism guarantees Pareto optimality in the commodity packages that will be "produced" and in the allocation of the elemental resources. The experimental results suggest that: (a) the procedures of the mechanism are operational, i.e., motivated individuals can execute the required task with a minimum of instruction and training; (b) the extent of demand underrevelation by participants is not large, i.e., allocative efficiencies of $98-99 \%$ of the possible surplus seem to be achievable over time with experienced bidders. This occurred despite repeated early attempts by inexperienced subjects to manipulate the mechanism and to engage in speculative purchases.

Subsequently, the idea of smart computer-assisted markets was applied in a proposal to deregulate the electric power industry in Arizona by separating the "wires business" from energy sales, and create a smart market in the form of the Arizona Energy Exchange, (Rassenti and Smith, 1986); in a Federal Energy Regulatory Commission study of the application of linear programming algorithms to the processing of node-specific bids to buy delivered gas and offers to sell wellhead gas, and leg-specific offers of pipeline capacity by multiple rights holders 
(McCabe, Rassenti and Smith, 1989); in an auction for payloads to be manifested on the Space Shuttle (Banks, Ledyard and Porter 1989); in a barter system for resources on the Cassini mission to Saturn (Ledyard, Porter and Rangel 1994); in an auction to schedule trains (Brewer and Plott 1996); in an auction for transportation services (Ledyard, Olson, Porter, Swanson and Torma (2000); in a two-sided combinatorial auction for trading pollution permits (Isikida, Ledyard, Olson and Porter, 2000); in a market to exchange financial portfolios (Bossarts, Fine and Ledyard 2000).

The RSB mechanism addressed three problems generic to the combinatorial features of the commodity space: (i) separating prices (Lagrange multipliers) in the optimization may not exist; (ii) in view of (i) what information should be reported to the bidders after each sealed bid auction allocation (or round in the case of multiple round auction mechanisms)? (iii) what are the behavioral incentive properties of the mechanism's rules? An integer programming algorithm was devised to allocate discrete elemental resources $\{0,1\}$ to the packages that maximize total surplus as reported in the bids submitted for the packages, subject to constraints on the supply of each elemental resource. Two pseudo-dual programs to this primal problem were used to define a set of accepted packages and a set of rejected packages; also a set of lower bound prices $\left\{w_{i}^{*}\right\}$ and a set of upper bound package prices $\left\{v_{i}^{*}\right\}$ were determined. Then, (a) if a package bid was greater than the sum of its component values in the set $\left\{v_{i}^{*}\right\}$, it was in the set of accepted packages, and, except in rare marginal cases, the bidder paid less for the package than her bid, providing good incentives not to under-reveal true value; (b) if it was less than the sum of its component prices in $\left\{w_{i}^{*}\right\}$ it was in the set of rejected packages; (c) all bids in between were in a region where acceptance or rejection were critically dependent upon the integer constraints on the allocation of the elemental resources. Thus, each bidder knew that in a subsequent auction (or round if an iterative procedure was used) whether a best reply would certainly be accepted, certainly be rejected, or depended on the integral "fitness" of the bid. ${ }^{5}$ A bulletin-board aftermarket was used to allow subjects to further adjust for elements needed to fill out packages after each primary auction using the combinatorial auction mechanism. This was compared with independent auctions in which each resource was auctioned simultaneously in a sealed bid uniform price auction, followed by the bulletin-board after-market. 


\section{$\underline{\text { Results }}$}

(1) This computational and feedback reporting process in RSB yielded efficiencies for experienced bidders no lower than $97.8 \%$ in a "difficult" combinatorial environment, and no lower than $83.2 \%$ in an "easy" combinatorial environment.

(2) Efficiency in the difficult environment with experienced subjects weakly dominated efficiency in the easy environment, and was consistently very high (98-99\%).

(3) In both environments efficiency in the RSB mechanism tended to be higher than the independent auction control mechanism.

(4) The lower efficiencies indicated in (2) and (3) were a consequence of subject attempts to strategically acquire units in the primary auction for resale in the after market. The independent auction invited these manipulative attempts much more prominently than RSB in both the easy and difficult combinatorial environments. With experienced subjects the RSB mechanism invited it slightly more in the easy than the difficult environment. This small difference was conjectured to be due to increased transparency when combinatorial complexity is minimal. In more complex environments it is especially costly to fail to obtain the desired packages: bidders are motivated to better reveal true package value and thereby rely on computer assistance to put together valuable packages. This was the primary learning experience that subjects went through in using the RSB mechanism. The fact that RSB performed better (relative to the independent auctions) in the more complex combinatorial environment is consistent with the findings reported in Banks, Ledyard and Porter and Ledyard, Porter and Rangel in which single-item commodity awards were less efficient than package-item awards. We suggest that the more transparent environments simply invite manipulation attempts more readily than complex environments where with private information it is more difficult to capture benefits that outweigh the cost risk of manipulation. The easiest way to control for gaming between the primary and after markets is to abolish the latter. The combinatorial auction performed so well in the primary auction that there was little room left for improvement in the after market.

\subsection{Implications for the Design of Spectrum Auctions}

Our discussion suggests several potential implications for the design of spectrum auctions, or similarly complex allocation problems. Auction design requires balancing a number 
of competing considerations, each one of which has an uncertain weight in the final specification of the mechanism to be used. Consequently, the implications listed here should be viewed primarily as a basis for further theoretical, experimental and field investigation, and for discussion among the growing number of scholars associated with applied mechanism design.

1. Virtually all the complexity of the SMA and its antecedents derives from the assumption that spectrum licenses are predominantly characterized by common, and/or affiliated, values, i.e. bidder values for licenses have strong common and/or positively correlated, component characteristics.

2. This assumption leads to the theoretical conclusion that revenue to the seller is greater if the identity of every bidder is made public in real time using the English auction format. The governing argument, originally, was based on an apocryphal metaphor of the form, "If AT\&T is bidding, their information is superior, and therefore what AT\&T is willing to pay is an indicator of value to others." By full revelation of private identities, the dispersed information on common value is pooled, enabling the price to fully reflect all such information. This assumption, we believe, has been accepted as an axiom without empirical examination or skeptical scientific challenge.

3. Even if the assumption has more or less validity, we believe the argument and theory based on it is flawed. Since the full information characteristics of the auction force revelation of value by the better-informed bidders, this undermines their original incentive to invest in acquiring information. To the extent, then, that there is under-investment in information that is not firm specific, this feature diminishes the importance of common values in the auction environment.

4. The stated primary objective of the FCC auctions is efficiency - the award of licenses to those who value them most and can most effectively employ them. But if bidder values are in truth nearly the same, an award to any bidder is efficient. Hence, there is a contradiction between the stated FCC objectives, and the assumption that licenses are dominated by commonality of value.

5. There exists no litmus test for common valuedness; i.e. we have no micro analytical procedure for going from the economic characteristics of the auctioned item to a statement about what proportion of a bidder's value is common to other bidders. 
6. Nor is there a procedure for solving this problem from observations on price data: we have a theorem (Jackson, 1999) showing that there is no equilibrium price for an item whose value is $\alpha$ percent private to bidder $i$, and $(1-\alpha)$ percent common to all bidders. This is because there is no way to infer from the bid alone what is the confounded mixture of common and private information that is reflected in that bid.

7. All these considerations call for laboratory and field tests of alternatives to the SMA and its derivatives (those allowing combinatorial bidding and unpublished bidder identities, including the sealed bid format). ${ }^{6}$

8. The examination of alternatives to the SMA, and its antecedents, is further indicated by the high transactions' cost of participation, requiring advice from a wide range of consultants to deal with the strategic issues that arise in real time as the bidding proceeds. The ideal incentive mechanism design should lead to a two step procedure: (1) an estimation of the value of the auctioned item(s), followed by (2) the submission of this value in the form of a bid, such action being a fair approximation to that which serves the interest of the bidder. Thus, in the combinatorial auction summarized above, which was only approximately incentive compatible, a difficult interdependent environment quickly yielded $98-99 \%$ efficiency with experienced bidders.

The key question for the SMA, and for combinatorial mechanisms based on open bidding, is the following: do the benefits, based on the judgement as to the importance of common valuedness, outweigh the costs of higher complexity induced by the desire to control manipulation. All the examples of strategic behavior problems - exposure, free rider, demand reduction, etc., - are based on postulated common information, known to the analyst and/or revealed in open English bidding. Consequently, the chain of causality is that common valuedness implies English format (with bidders publicly identified) implies strategic behavior implies higher transaction cost design complexity to control that behavior. This chain needs fundamental examination.

The SMA has evolved over a sequence of field applications in which weaknesses and defects revealed in each application led to "fine tuning," followed by the observation of further problems leading to new "fixes," and so on. Each "fix," designed to limit strategic exploitation, tended also to generate complexity and its attendant higher transactions' cost. This is reminiscent of the MRS series of experiments leading to a sequence of increasingly complicated 
modifications of English procedures until all such fine tuning attempts were abandoned in favor of the elimination of price bidding - the feature which historically had been the defining characteristic of the English auction - and quoting prices by an English Clock.

9. In the spectrum auctions probably very little of the ultimate technological, antitrust and demand uncertainty is resolved at the end of the auction. This feature is not formally part of the theory that led to the original conclusion that the English auction procedure should be used. But whatever might be the consequence of reexamining the basis for the SMA in the light of this feature, a more important consequence, we think, is the challenge to the assumption that these licenses should be privatized by a single initial one-sided auction. More appropriate, we think, is for the FCC to consider a periodic two-sided auction exchange mechanism that would facilitate repackaging of old as well as new rights in response to changing information and technology. Whatever the format chosen, allowing all spectrum title holders to participate in the sale of incumbent rights in each new auction addresses misallocations caused by bidding errors or various mechanism design faults in earlier auctions, together with subsequent resolution of earlier uncertainties about technology, demand and antitrust.

10. If open English bidding continues to be the FCC's procedure of choice, we believe it should eliminate iterative bidding by the participants. Rather, all prices should be supplied by English Clocks, with bidders simply indicating whether they remain active, or not. This is a straightforward method for auctioning goods independently, which is the environment to which the FCC's SMA applies. Such an auction does not require a bidder to be present during its course. For a good of interest, the bidder need only submit a benchmark price below which his bid is tendered and above which his bid vanishes.

\section{Part 2: An Experimental Analysis of the Federal Communications Commission's Eligibility Rules for Spectrum Auctions}

\subsection{Unequal Eligibility Points and Tapered Eligibility}

A market institution consists, in part, of a set of explicit rules that govern the actions and behavior of the market participants. Because of the complexity of the expected bidding environment and the potential for strategic behavior on the part of participants, the SMA employed by the FCC includes numerous rules. We examine the effects of two SMA rules - the rule that assigns unequal eligibility points to different licenses, and the rule that tapers the 
amount of eligibility that the bidder maintains during the course of the auction for a given level of bidding activity.

\subsubsection{Eligibility Rules}

During the SMA, participants submit single-item bids for individual spectrum licenses. Once the bids are submitted, the current high bids and the current high bidders for each license are identified. Participants have the opportunity to raise their bids in subsequent rounds. The auction is a "simultaneous" auction in that all licenses are put up for sale concurrently and the individual auctions for the separate licenses all close at the same time. The simultaneous and iterative nature of the SMA raises the issue of how best to control the speed with which the auction progresses. It is possible that in some of the spectrum auctions bidders benefit from knowing their rivals' bidding strategies and license value estimates. Consequently, bidders may have the incentive to limit their bidding activity and simply observe their rivals' bids. To address this concern, the FCC adopted "eligibility rules." These rules encourage early and continued bidding by limiting a bidder's ability to bid on licenses in subsequent bidding rounds if he fails to maintain a certain level of bidding activity in the previous round.

To establish eligibility conditions the FCC considers the product of the amount of spectrum (megahertz) assigned to a given license and the total population within the license service area. (See section 2.1.3 below for how our environment parameterizes population effects). This "MHz-POPs" value for each license is assigned a number of eligibility points. A bidder maintains eligibility points for bidding in subsequent rounds by owning the current high bid on a license, or by submitting an "improving" bid that is equal to the current high bid plus some specified bid increment. Because a bidder's eligibility points determine his bidding flexibility, a bidder should assign an "option value" to the maintenance of such points.

Believing that bidders may need substantial bidding flexibility in the early rounds of the auction, the SMA employs a multiple stage, tapered eligibility rule. Each stage is defined according to the number of eligibility points bidders obtained when bidding on a particular license times a scalar greater than $1 .^{7}$ Recognizing that some of the bidding activity may be motivated by strategic considerations, the SMA attempts to restrict bidding flexibility in later rounds. It accomplishes this goal by successively reducing the scalar from stage to stage. For 
example, a reduction in the scalar factor from 1.5 to 1.0 effectively reduces by one-third the number of eligibility points a bidder can maintain by bidding on a particular license.

Bykowsky and Cull (1998) note that the inequality in the number of eligibility points derived from bidding on spectrum licenses means that, for a given bidder, the size of the option value derived from bidding on a license: (1) differs across licenses; and (2) increases as the eligibility tapers. The inequality in option value across licenses may result in artificially high prices for licenses with "low" population (e.g., low eligibility points) and a less efficient license assignment. This result would occur if high valued bidders failed to maintain sufficient bidding flexibility in later rounds to be able to bid on licenses that cover "large" populations. Milgrom(2000) coined the term eligibility "parking" to describe a strategy in which bidders disguise true value for certain licenses by bidding on other licenses which are not as valuable at current auction prices. For whatever reason a bidder may wish to do so, inequality in eligibility points was thought to make the adoption of a "hide in the weeds" strategy more difficult, thus enhancing efficiency by reducing strategic behavior.

Eligibility management has been identified as a major strategic concern of spectrum auction participants (see Salant (1995)). Flexible eligibility (relaxing the eligibility constraint rule by always using a scalar greater than 1) may enhance assignment efficiency by increasing a bidder's ability to switch to (a set of) higher point licenses in a round when their profit margins have become relatively larger. Moreover, flexible eligibility may be most effective in the presence of unequal eligibility points, as that condition increases the difficulty in managing eligibility. However flexible eligibility may prolong the length of the auction.

In summary, the introduction of eligibility point budgets, designed to control strategic behavior, leads to new strategic behavior (parking), which in turn leads to flexible eligibility, which, if excessive, may prolong the auction. Our experiments are designed to include an examination of the last implication due to this chain of design modifications.

\subsection{Experimental Design and Protocols}

This section describes in detail the four different eligibility rules used to run the SMA experiments, the protocols employed to train subjects, subject payoff incentives, methods for dealing with individual bankruptcy, subject recruitment, the nature of the licenses auctioned, and the structure of the valuations possessed by bidders. 


\subsubsection{Auction Rules}

In these SMA experiments ten licenses were offered for sale. The licenses were generically labeled A, B, C,..., J. Participants submitted a series of single-item, sealed bids for desired licenses. Following the submission of such bids, the high bid for each license is posted. These high bids then become the standing bids for the next round of bidding. In addition to these general rules, we adopted the following specific rules:

0. Activity: To be able to submit a bid in a round, a participant must have submitted an acceptable bid in the previous round or have had a standing bid two rounds previous.

1. Acceptable bids: For a bid to be acceptable in any round, it must be greater, by a prespecified increment, than the standing bid for that license.

2. Bid increments: The minimum acceptable bid for a license in the next round is the current standing bid plus a fixed percentage of that standing bid. The fixed percentage is determined by the excess bids placed on the license. Specifically, if one bid was submitted on the license the minimum acceptable bid would be $(1+.05)$ times (the standing bid); if two bids were submitted on the license then the minimum acceptable bid would be $(1+.10)$ times (the standing bid); if three or more bids were submitted on the license then the minimum acceptable bid would be (1+.15) times (the standing bid). ${ }^{8}$

3. Bid Withdrawal: Subjects are allowed to withdraw any of their standing bids before a round begins. After a withdrawal, the FCC becomes the standing bidder for the withdrawn license and replaces the bid with one that is less than or equal to the withdrawn bid. An individual who withdraws a bid pays a penalty equal to the maximum of zero or the difference between the amount of the bid he withdrew and the highest bid submitted by a participant other than the FCC after his withdrawal. ${ }^{9}$ In the past, the FCC did not limit the number of withdrawals a participant could use. With concurrence from the Federal Communications Commission, we used a limit of 2 rounds of withdrawals per subject, ${ }^{10}$ which is their current rule.

4. Eligibility: Associated with each license is a fixed number of eligibility points. A participant is constrained to bid for at most a subset $\mathrm{S}$ of licenses, other than the ones she has the current standing bid on, that satisfies the following eligibility constraint: 
$\left(\sum_{i \in S}\right.$ Points $\left._{i}\right) \leq$ (the sum of the eligibility points of licenses for which the participant submitted acceptable bids in the previous round and does not currently have the standing bid + the sum of the eligibility points of licenses for which the participant had the standing bid 2 rounds previous but no longer has the standing bid)

5. Stopping rule: The auction stops when all bidders have no eligibility remaining beyond their standing bids. The items are awarded to the participants with the standing bids and any withdrawal penalties are computed and paid at that time.

In a departure from the currently used SMA, the tested SMA did not include bid waivers and an up-front deposit rule. A waiver allows a bidder to extend to the next subsequent bidding round its current level of bidding eligibility without bidding in the current round. A bidder may desire such flexibility in response to either a computer hardware or software problem, or due to an unexpected need to consult with senior management or paid advisors on bidding or financing matters. However, given that these situations were not part of the experimental environment, a waiver rule was not a necessary element of the tested SMA. ${ }^{11}$

The SMA also requires that a participant make a "significant" upfront deposit. The upfront deposit determines the maximum number of eligibility points a bidder can attempt to acquire in any given round. Since we did not impose budget constraints on participants in the experimental environment, there was no need for initial deposits. Instead, we simply allowed each bidder to be eligible to bid on all licenses in the first round.

\subsubsection{Treatment Variables and Structure}

\subsubsection{Flexible Versus Nonflexible Eligibility}

The first treatment variable took on two discrete values: Flexible and Nonflexible. To create the two treatments, the right-hand side of the inequality defined in rule 4 above, is simply multiplied by a scalar $\omega \geq 1$.

In the Flexible treatment $\omega=1.5$ and in the Nonflexible treatment $\omega=1$ for all bidding rounds. ${ }^{12}$ 


\subsubsection{Unequal Versus Equal License Eligibility Points}

The second treatment variable also took on two discrete values: Unequal and Equal. In the Unequal treatment each license was assigned a specific number of eligibility points (recall that eligibility points in the SMA are based upon the size of population covered by the license and the amount of spectrum-megahertz in the license). Table 1 shows the assignment of eligibility points for each license in the Unequal condition.

In the Equal treatment each license was assigned exactly1 point.

\subsubsection{The Structure of Treatment Variables}

Given that each treatment variable takes on two values, there are four different rule treatment combinations. Table 2 presents these four combinations. Experiments comparing the four different eligibility rule treatments will permit an examination of the potential tradeoff between, for example, greater assignment efficiency and prolonged auction length.

\subsubsection{Market Demand}

The effect of a particular eligibility rule treatment on observed outcomes may depend upon the bidding environment. The bidding environment is characterized by the level of superadditivity among license values, whether or not a competitive equilibrium exists, and the difference in values between the highest and second highest valuations, among other factors.

\subsubsection{Valuation Structure}

Economic theory (Bykowsky, Cull, and Ledyard (1999)) and past experiments (Ledyard, Porter and Rangel (1998)) suggest that the SMA should have little problem in generating

efficient allocations when bidder valuations are additive. ${ }^{13}$ In such an environment, the SMA should produce a set of prices and a license allocation that closely approximate the results obtained under competitive equilibrium conditions. However, problems may arise when bidder valuations exhibit superadditivities or synergies (we will use these terms synonymously for the remainder of this paper). ${ }^{14}$ 
In our experiments, subject i's valuation $\left(\mathrm{V}^{\mathrm{i}}(\mathrm{X})\right)$ for a subset of licenses $(\mathrm{X})$ is parameterized by the following expression:

$$
\begin{gathered}
\mathrm{V}^{\mathrm{i}}(\mathrm{X})=\Sigma_{\mathrm{j} \in \mathrm{X}} \mathrm{V}_{\mathrm{j}}^{\mathrm{i}}+\lambda^{\mathrm{i}}\left(\Sigma_{\mathrm{j} \in \mathrm{X}} \mathrm{q}\right)^{\beta \mathrm{i}}+\Delta^{\mathrm{i}}\left(\Sigma_{\mathrm{j} \in \mathrm{X}} \Sigma_{\mathrm{k} \in \cup \mathrm{A}^{\mathrm{j}}} \delta^{\mathrm{j}}(\mathrm{k})\right)^{\alpha \mathrm{i}} \\
\text { where } \lambda^{\mathrm{i}}, \beta_{\mathrm{i}}, \Delta^{\mathrm{i}} \text { and } \alpha_{\mathrm{i}} \geq 0 .
\end{gathered}
$$

The first term represents the sum of the stand-alone values of the licenses in the subset L. ${ }^{15}$ The next two terms were added to model two potential license value superadditivities. The second term captures a scale economy of population $(q)$ that arises from using existing billing service and maintenance departments with the new service. ${ }^{16}$ The reduction in the average total service cost per customer introduces a superadditivity in a bidder's license valuations for a given set of licenses. The third term attempts to capture license value superadditivity that results from the value a mobile service subscriber places on being able to roam seamlessly from one service area to another. Due to this effect, some bidders may experience a superadditivity in license valuation across a set of contiguous licenses. To represent this type of value superadditivity, consider the topology of licenses described in Figure 1 below. Each point on the wheel represents a license and a geographical location. Licenses adjacent to each other (e.g., license $\{1,2\}$ and $\{8,1\})$ are considered contiguous. The set of licenses contiguous to any licenses is given by the set $A^{i}$ in the value equation above. $\delta^{j}(K)$ is the indicator function that equals 1 for each contiguous license.

Experiments were conducted under four different valuation environments: (1) additive; (2) low superadditivity; (3) medium superadditivity; and (4) high superadditivity. The four environments were generated by varying the parameters, $\lambda, \beta, \Delta$, and $\alpha$, the separate increases in which, lead to an increase in the degree of license value superadditivity. Table 3 shows the parameters used in each environment:

In addition to the conditions established by the parameter values, we also varied other characteristics of the bidding environment that may, according to Charles River and Associates (See Charles River and Associates Report 1B (1998) pages 5-7), create performance difficulties for the SMA auction. These other characteristics were:

1. Number of bidders

2. Existence of a Competitive Equilibrium $(\mathrm{CE})^{17}$

3. Package Overlap ${ }^{18}$ 


\subsubsection{Subject Pool and Procedures}

A total of 150 subjects were recruited from upper-class courses in accounting, information systems, economics and engineering at the University of Arizona. Each subject was trained in the auction rules through a series of 3 two-hour experimental sessions in which withdrawal rules, activity rules and synergy values were added sequentially to the mechanism and environment. Following the third training step, subjects were divided into two separate groups. One subject group trained exclusively in bidding environments where licenses were assigned unequal eligibility points, while another subject group trained exclusively in bidding environments where licenses were assigned equal eligibility points. Separating subjects in this manner eliminated any possibility that subjects may perform less effectively due to confusion regarding the adopted eligibility rule.

\subsubsection{Bankruptcy}

Economic theory suggests that winning bidders may end up losing money in an SMA in the presence of superadditivities in valuations. Consequently, we incorporated a bankruptcy rule within the SMA. Under this rule, which was read aloud to the subjects prior to the experiments, each subject was given $\$ 15$ at the start of the experiment. If, following the completion of any particular auction within the experiment, a subject's total losses exceeded $\$ 15$ plus his earnings so far, the subject was considered financially bankrupt and prohibited from continuing to participate in the experiment.

\subsubsection{Design Summary and Procedures}

Table 3.6 in Appendix A, lists the conducted experiments. An experiment includes a series of SMAs. Subjects are assigned different valuations across the auctions. Each auction involved the sale of ten licenses labeled A through J. Superadditive valuations applied within two distinct license sets; Set $\Phi=(\mathrm{A}, \mathrm{B}, \mathrm{C}, \mathrm{D}$, and $\mathrm{E})$ and Set $\Psi=(\mathrm{F}, \mathrm{G}, \mathrm{H}, \mathrm{I}$ and $\mathrm{J}) .{ }^{19}$ Each set was assigned either an additive, or low, medium, or high superadditive value environment. For example, in the first experiment involving the Baseline treatment Set $\Phi$ had an additive value environment while Set $\Psi$ had a high superadditive environment. Instructions for each of the treatments can be accessed at http://linus.econlab.arizona.edu/FCC_Instructions, the experimental 
parameters used can be found at http://linus.econlab.arizona.edu/FCC_Parameters and the data for each experiment can be found at http://linus.econlab.arizona.edu/FCC_data.

\subsection{Experimental Results}

Congress has mandated that the FCC consider assignment efficiency as the primary measure of performance when evaluating the desirability of using a particular auction mechanism for assigning spectrum licenses. A secondary factor to be considered is the revenue that will be generated by the auction. In addition to these two criteria, we also measured the length of time, expressed in rounds, the auction took to close, and whether winning bidders incurred substantial losses from acquiring their licenses. Auction duration and bidder loss measures have important practical significance. Auctions that take a long time to close impose a heavy transactions cost upon participants. Similarly, heavy losses by bidders may result in the re-auctioning of the acquired licenses. The re-auctioning of the license extends the period of license non-use and, in so doing, deprives society of important benefits. Finally, we examine the frequency with which participants withdraw high bids. In some environments, a high number of withdrawn bids indicates that bidders are having substantial difficulty in obtaining their most desired licenses.

The goal of the statistical analysis is to draw inferences on the change in assignment efficiency, auction revenue, bidder losses, withdrawals, bankruptcies, and auction length by auction mechanism and bidding environment. Efficiency, revenue, losses are modeled with a linear regression model. Withdrawals, bankruptcies and auction length are modeled with a linear Poisson model. Moreover, this analysis will be conducted on data generated from distinct bidding environments - additive valuations versus superadditive valuations. ${ }^{20}$ In addition, we supply Tables and Figures showing the mean and/or median measure of efficiency, revenue, etc. to give the reader an indication of the magnitude or importance of the effect of each treatment value.

\subsubsection{Efficiency}

An auction is said to be $100 \%$ efficient when it assigns the set of offered items so that the total value that society obtains from the items is maximized. An auction mechanism's ability to efficiently assign items is measured as the ratio of the sum of the values that assignees place on their items divided by the sum of the value-maximizing assignment. Efficiency of less than 
$100 \%$ can only occur when at least one bidder fails to make a purchase that is contained in the optimal allocation.

\subsubsection{Additive Environment}

Table 4 shows the average level of assignment efficiency of the SMA auction under the additive environment for all discrete treatment variable combinations. Recall that each experiment had two groups of 5 licenses to be allocated in each auction. Each group in the "environment pairing" had a different value structure. The efficiency below is averaged over all groups of five additive licenses.

Result 1: Replicating previous studies, the additive environment generates almost perfectly efficient outcomes with no statistically significant difference across the treatments.

Table 4 shows the mean and median efficiency for each treatment. In addition, Table 5 shows the count of $100 \%$ outcomes in each treatment. In the baseline additive environment $60 \%$ of the experimental outcomes are at the $100 \%$ level of efficiency.

Table 6 provides the parameter estimates for the analysis of efficiency in the additive environment.

Table 6 shows that in the additive environment neither treatment (Flexible nor Unequal) has any significant effect on efficiency, nor does their joint occurrence. (An indicator variable that accounts for the interaction of two variables will be named using the symbol $\wedge$ in this and following tables.) In addition, it shows that there is no significant effect from the number of bidders in the auction or from pairing the additive environment with either a high or medium synergy environment.

The largest number of non-100\% outcomes occurs in the treatments in which Unequal eligibility points were used. If subjects were using licenses for option values we would suspect that licenses with the highest eligibility points would be the ones misallocated. This cannot be confirmed by the data (see figure below). 


\subsubsection{Superadditive Environment}

We conducted experiments with various levels of superadditivity - low, medium, and high - associated with each subgroup of 5 licenses. Table 7 provides the mean, (median) efficiency for all of the superadditive environments:

In addition to the superadditive value structure of each group of 5 licenses, we assigned three other properties to each group:

1. The number of bidders. ${ }^{21}$

2. Whether there exists a competitive equilibrium (CE) set of prices for each license at which there would be no excess demand for any license and the efficient allocation is obtained.

3. The degree of Overlap among packages. In each environment we selected the average size of the packages that had superadditive values assigned to bidders: the greater the average number of licenses in a package, the greater the overlap.

The effects of the three properties above are included in Table 9, from which we conclude that:

Result 2: The Flexible treatment has a positive and statistically significant effect on assignment efficiency. Overlap has a statistically significant negative effect on efficiency. All other variables are statistically insignificant.

Thus we find that under superadditive value conditions, flexibility provides an important boost to efficiency, while increased density of package overlaps tends to decrease efficiency.

\subsubsection{Revenue}

The amount of revenue generated from a particular auction mechanism depends, in part, on the distribution of license valuations across active bidders. This distribution is altered by changes in the number of bidders in the auction: for example, when financial difficulties occur and a bidder decides or is forced to leave. One method of controlling for the confounding effect of changes in the number of bidders on auction revenue is simply to "normalize" auction revenue 
on the basis of what could be realized given the set of remaining participating bidders and their valuations.

\subsubsection{Additive Environment}

In the additive environment analysis, we use the ratio of revenue actually collected divided by the sum of the competitive equilibrium license prices for the remaining agents.

It is easy to see from Table 10 that revenue is substantially above the competitive equilibrium predictions. We will discuss this over-bidding phenomenon in section 4.3 below.

As we did with efficiency we estimate the ANOVA model using the normalized revenues generated from the observed auction outcomes.

The ANOVA substantiates the following generic result:

Result 3: There is no statistically significant difference in revenue among the treatments in the additive value environment.

\subsubsection{Superadditive Environment}

In the superadditive environment analysis, we normalize "revenue" as the ratio of the maximum total value assignment for the remaining agents divided by the revenue actually collected. Table 12 shows these (mean, median) normalized revenue ratios:

The related ANOVA presented in Table 13 substantiates the following result:

Result 4: Only the auction rule treatment have a significant effect on revenue.

It seems that revenue in the superadditive case is very sensitive. A rule that specifies Unequal points provides lower mean revenue (0.72) than one that specifies Equal points (0.92). A Flexible eligibility rule provides higher mean revenues $(0.98)$ than a Nonflexible rule $(0.84)$.

\subsubsection{Competitive Equilibrium Predictions}

A set of competitive equilibrium (CE) prices exists in all the additive value environments and in some of the superadditive value environments. We now check to see if these CE prices are good predictors of the final bids. Because of the use of click-box bidding, the CE prices would be a good predictor of final prices if the latter were within $15 \%$ of the former. ${ }^{22}$ Figure 3 
below shows the box-plot of the relative price outcomes (highest bid / CE price) across each treatment for the additive value environment. In the box plot the dark points shows the medians, the rectangles show the range of the two middle quartiles, and the fences show the range of all other outcomes, with the exception of any outliers which are less than $2 / 3$ or greater than $3 / 2$ of the median. Outliers are shown as open circles.

In every case in Figure 3, the median ratio of highest bid to CE price exceeds unity and in a significant number of instances a winning bid exceeds the maximum value of the $\mathrm{CE}$ prediction. In fact the high bid distributions are often uniformly above the CE price. Ubiquitous jump bidding appears to be the principle culprit, although in a few instances these price discrepancies can be traced to bidders "parking" in licenses (making negative earnings in at least one round on a license).

Figure 4 below is a box-plot of the relative price outcomes (highest bid / CE price) across treatments for the superadditive value environment when a set of CE prices exists. Figure 4 shows winning prices are often lower than the $\mathrm{CE}$ range of prices.

Figures 3 and 4 strongly suggest the following result:

Result 5: Competitive equilibrium prices are poor predictors of bidding behavior in an SMA.

\subsubsection{Bidding Rounds}

One proposed factor in evaluating auctions is their duration. A reasonable assumption is that longer auctions should be avoided ceteris paribus. This reduces the transactions cost incurred by buyers and the sellers, and thus potentially raises effective valuations and net revenues. Recall that our experiments consisted of 4 different value environments. Within these environments we can determine the treatment effects on rounds. These are presented below in Table 14:

It comes as little surprise that, in each environment, a Flexible bidding eligibility rule extends the length of the auction. Table 15 below verifies the statistical significance of the observed outcomes in Table 14 and qualifies the following result.

Result 6: In these experiments when the points scalar is increased from 1.0 to 1.5, the number of rounds in the auction significantly increases. 


\subsubsection{Bidder Losses}

It may be in the interest of the auctioneer to minimize bidder losses. This need may arise from a desire to accommodate the budget constraints of smaller buyers. An auction that involves the risk of loss may bias auction participation toward larger bidders who can risk small potential losses for the possibility of a large gain. Table 16 shows the average losses for each treatment in the additive case. These averages come from exactly two losses in the 165 markets (33 sessions).

Result 7: In the additive markets there are virtually no losses.

However things are considerably different when synergies are involved. Table 17 shows that losses occur frequently in superadditive environments. In approximately $80 \%$ of all auctions across all the treatments there was a loss by at least one bidder. The large average loss in the highly superadditive case with no points and tapering is skewed by two bankruptcies of $\$ 102$ and $\$ 64$.

The ANOVA results show that at this point we can do no better than speculate that it is the SMA institution itself that is at fault in causing bidder losses, since none of the factors examined appear to be the cause. However, it may be the case that in the bidding environments construed, no auction institution would have performed well in this regard. This possibility remains to be investigated.

Result 8: Though losses are an important and common occurrence in an SMA when values have synergies, none of the treatment variables examined provide individually compelling explanations for those losses.

\subsubsection{Bankruptcies}

In our experiments bidders participated in a sequence of independent auctions. It was possible for a bidder to incur losses large enough in one auction so that his earnings and working capital were eliminated. In this case the bidder was considered bankrupt, was asked to leave the 
experiment, and was not able to participate in subsequent auctions during that experimental session. Table 19 shows the number of bankruptcies per auction and treatment.

Although Table 20 provides no significant evidence that the number of bankruptcies in any of the superadditive environments is statistically different from 0 , we may simply observe from Table 19 that:

Result 9: Bankruptcy never occurred in the additive environment and had the highest aggregate frequency in the medium synergy environment.

\subsubsection{Withdrawals and Combined Withdrawal and Bidding}

One option available to bidders in the SMA is to withdraw a standing bid. Table 21 shows the number of withdrawals per auction. Across all treatments and environments the average number of withdrawals per auction was less than 1 . Though the average number of withdrawals was few, the fewest number predictably occurred with additive valuations while the largest number occurred when synergies were high. Table 22, which provides a Poisson model of number of withdrawals for all treatments in the superadditive environments, suggests that, of the treatments considered, only Overlap conditions increased the probability of a withdrawal.

To speed-up the SMA, Charles River and Associates Report 1A (1998) pgs 3-4 and 8-9 recommended combining the withdrawal and bidding portion of the auction in each round. The implementation of this rule, as defined by the FCC, requires that the bidder who is withdrawing a bid cannot also submit a bid on that license in the round in which he is withdrawing it. During our experiments we observed many cases in which withdrawals occurred late in the auction as participants were shedding eligibility. In over $50 \%$ of the cases in which a withdrawal occurred, the individual withdrawing did not know that others were simultaneously reducing their eligibility. The end effect was that these individuals gave up licenses that no one else picked up: the license reverted to the auctioneer and the withdrawing individual paid the full withdrawal penalty.

Table 23 shows, for the superadditive environments, the number of licenses that went unsold at the end of the auction due to withdrawal as a fraction of the number of total withdrawals. Every withdrawal tendered in an auction occurred when the bidder was losing 
money from a failed aggregation. We could not detect any use of withdrawals for strategic reasons like those suggested in the Charles River and Associates report.

We conclude that it may be very important to allow bidders to bid on a round before withdrawing.

Result 10: Environments with high synergy will always produce withdrawals in an SMA. Many of those withdrawals will occur toward the end of the auction, and approximately 33\% will remain unsold often because a potential buyer gives up eligibility in the same round without knowing that the license he desires will become available in the next round at a much lower price.

\section{Part 3: An Experimental Comparison of the Simultaneous Multi-round Auction (SMA) and a Combinatorial Multi-round Auction (CMA) to Allocate Spectrum}

\subsection{The Charles River and Associates' Proposed Combinatorial Multi-round Auction}

Charles River and Associates was contracted by the FCC to design a combinatorial mechanism to allocate spectrum. We will refer to that mechanism as the Combinatorial Multiround Auction or CMA. As with the SMA, the CMA proceeds in rounds. In each round, participants can submit a series of sealed single-item and/or packaged bids. ${ }^{23}$ Following the submission of such bids, an integer programming algorithm finds the set of value maximizing bids such that each license is allocated to only one participant and all package constraints are not violated. Winning bids are posted for all to see along with the best single license bids. All of the bids submitted in the current round are used to "constrain" the bids that can be submitted in the next round. The constraints are determined using the following specific rules.

0. Activity: In order to be able to submit a bid in a round, a participant must have submitted an acceptable bid in the previous round or have had a standing bid two rounds previous.

1. Acceptable bids: In order for a bid to be acceptable in any round, it must be 5\% greater than the best combination of bids, from the previous round, which exactly span the licenses in the bid.

2. Bid Cancellation: Bids from previous rounds, whether they are winning bids or not, are automatically submitted in the next round and count against eligibility unless they are removed or canceled from the system. ${ }^{24}$ 
3. Eligibility: Each license has the same fixed number of eligibility points associated with it, namely 1 . Participants cannot bid for more licenses than they have eligibility points. Eligibility has two rules. The first rule is that the union of all bids (that is the number of distinct licenses bid on, whether they are in packages or singles) cannot exceed a bidder's eligibility. The second rule is that the sum of the number of licenses in packages (for all packages of two items or greater) cannot exceed a bidder's eligibility. Thus, if a participant has 5 eligibility points, he can bid on any five single licenses AND also place package bids as long as the number of licenses in all packages submitted is less than or equal to five. Eligibility in the next period is the minimum of the participant's eligibility in the previous round or the activity in the current round which is defined by:

$\lambda \bullet\left(\beta_{1} \bullet\left[\right.\right.$ number of licenses you are currently winning] $+\beta_{2} \bullet$ [number of licenses in bids that are not winners but meet or exceed the minimum increment $]+\beta_{3} \bullet[$ the number of licenses that are not in the winning set or meet the minimum increment but are above the best single item bids]

In our experiments we set $\lambda=3$; and $\beta_{1}=\beta_{2}=\beta_{3}=1$.

4. Stopping rule: The auction stops when no new acceptable bids are submitted. ${ }^{25}$

5. Information: All bids submitted or left in the system by all other participants are revealed after every round.

\subsection{Experimental Design}

The market demand values are exactly those employed in analyzing the SMA in Part 2. The only procedural difference between the CMA and SMA was the amount of time each experiment was allotted. We discovered that the CMA mechanism took substantially longer to run and extended the experimental sessions to 2.5 hours.

We recruited 100 subjects from upper-class courses in accounting, information systems, economics and engineering at the University of Arizona during the Fall 1999 semester. Subjects participated in two, $21 / 2$ hour experimental sessions in which they were introduced to a progression of more complicated combinatorial auction rules. Subjects could not participate in the full CMA until they had completed the 5 hours of training. 


\subsubsection{Design Summary and Procedures}

Table A1 in Appendix A lists all SMA and CMA experiments. The CMA parameter designs mimicked those used for the SMA. Subjects were assigned different valuations across the auctions. Each auction involved the sale of ten licenses (i.e., A through J). Superadditive valuations applied to various subsets of two independent license sets; Set $\Phi=(\mathrm{A}, \mathrm{B}, \mathrm{C}, \mathrm{D}$, and E) and Set $\Psi=(\mathrm{F}, \mathrm{G}, \mathrm{H}, \mathrm{I}$ and $\mathrm{J}){ }^{26}$ Each set was assigned either an additive, or low, medium, or high superadditive value environment. For example, in the first experiment involving the Baseline treatment Set $\Phi$ had an additive value environment while Set $\Psi$ had a high superadditive environment. Instructions, experimental parameters, and data for each CMA experiment can be found at the web sites listed in section 2.1.6. ${ }^{27}$

\subsection{Comparative Experimental Results}

We will compare the SMA and CMA on the basis of the criteria established to measure the performance of the SMA as described in Part 2.

\subsubsection{Efficiency (as defined as in section 2.2.1 above)}

\subsubsection{Additive Environment}

Table 24 shows the average (and median) level of assignment efficiency of the SMA and CMA for the additive environment. Table 25 shows the number of $100 \%$ outcomes from the total number of experiments conducted.

Result 11: The CMA is slightly more efficient than the SMA in the additive environment but both generate very high efficiencies.

Table 24 shows a mean difference of $2.6 \%$ and has a rank sum test statistic of $Z=1.42$ which is significant at the $10 \%$ level of confidence. In Part 2 we reported that having more flexibility increases SMA efficiency. Thus, we conjecture that much of the above difference in efficiency stems from the fact that the CMA was more generous in its flexibility than the SMA as the test implementation used a rule of 3 times activity to determine eligibility. We also point out that although bidders are free to submit packages in the additive environment they rarely do so. Fewer than $2 \%$ of all bids are package bids in the additive environment. 


\subsubsection{Superadditive Environment}

Table 26 provides the mean (median) efficiency for various levels of superadditivity low, medium, and high - associated with each subgroup of 5 licenses.

Result 12: In every superadditive environment the CMA outperforms the SMA with average efficiency gains ranging from 16\% - 22\%. The largest gain occurs in the high synergy case.

The rank sum statistics for the superadditive environments are $Z=9.76$ (low), $Z=8.75$ (medium), and $Z=12.36$ (high) which are all significant at less than 1\%. In Figures 5a-c we supply the distributions of efficiencies for each superadditive value environment.

\subsubsection{Revenue}

As in section 2.2.2 above we "normalize" auction revenue on the basis of what could be realized given the set of remaining bidders and their valuations after any bankruptcies.

\subsubsection{Additive Environment}

Table 27 shows the mean (median) normalized revenue ratios and suggests the following result:

Result 13: Revenue is significantly above the competitive equilibrium predictions. Revenue is higher in the SMA than the CMA.

There is a significant amount of jump bidding (above minimum increment requirements) in both the SMA and CMA, which causes the revenue to exceed competitive expectations. One reason that revenue may be even higher in the SMA is that bids had to be in incremental jumps $(5 \%, 10 \%$ or $15 \%)$ while in the CMA they simply had to be any number above $105 \%$ of the standing bid. 


\subsubsection{Superadditive Environment}

In the superadditive environment analysis, we normalize "revenue" as the ratio of the maximum total value assignment for the remaining agents divided by the revenue actually collected. Table 28 shows the mean (median) normalized revenue ratios:

Result 14: In the Superadditive environments, the SMA generates more revenue than the CMA with the largest difference coming in the high synergy case.

The rank sum statistics for these ratios are $Z=3.98$ (low); $Z=4.63$ (medium); and $Z=8.78$ (high), which are all highly significant. This result coupled with the efficiency results may seem to be a puzzle: how can the SMA, which is less efficient than the CMA, generate significantly more revenue? The main reason for the divergence is that there are no losses by bidders in the CMA, while in the SMA, losses because of failed license aggregations ranged from $4 \%$ to $35 \%$

of the revenue raised. These external losses are not part of measured efficiency ${ }^{28}$ that simply compares the total social value of realized and optimal allocations and is not concerned with participant and auctioneer division of surplus. Hence, the lower revenue from the CMA is not a disadvantage, given the FCC's efficiency objectives, because the CMA facilitates license aggregations and enables the participants to avoid losses, compared with SMA. Similar effects were reported by Rassenti, Smith and Bulfin in the first combinatorial auction study.

\subsubsection{Bidding Rounds}

In consultation with the FCC and the Charles River and Associates' CMA designers, the amount of time allowed for bidding in each auction round was increased for the CMA versus the SMA due to the complex nature and potentially large number of bids that the participants might tender in the former. We list the mean and median rounds required to complete each auction in Table 29.

Result 15: The CMA auction takes more than 3 times as long as the SMA to finish.

It should be noted, however, that more than $60 \%$ of the CMAs went for as many as 20 rounds without the realized allocation or revenue changing before the auction finally closed. 
There is clearly some opportunity to create a revised implementation that would curtail this wheel spinning.

In summary, these baseline experiments indicate that when the CMA is used, efficiency is increased between $2.6 \%$ and $22 \%$, but auction duration is extended considerably.

\subsubsection{Boundary Experiments}

Given the success of the Charles River and Associates' CMA in generating high efficiencies in the baseline superadditive environments originally tested, we created several additional environments that we thought might put pressure on both auction mechanisms. Such "stress tests" have become a fairly standard part of experimental methodology, particularly in applied economic design problems. After experimental results are reported, clients will often want to see a mechanism pushed to the edge of validity for some principal finding. Following the examples found in Charles River and Associates (1998), we focused on the threshold problem presented in their report. In particular, we developed two measures that might influence the ability of the Charles River and Associates' CMA to overcome the threshold problem. Recall that the threshold problem occurs when a large package of licenses bid on by a single bidder must be displaced by a group of 'small' bidders bidding on subsets of that large package. The 'small' bidders must then come to some agreement, through the mechanism, on how much each will contribute to overcome the large bid.

\subsubsection{Boundary Case Experiments}

Two environmental conditions were devised to stress the two auction mechanisms:

Condition 1: The Gain Effect. Gain measures the relative value of the optimal allocation ( $\left.\mathrm{V}^{*}\right)$, which is purposefully composed of several small bidder packages, and the next highest value allocation ( $¥)$, which is constructed to be a single bidder's value for the large package covering an optimal set of packages. We define the Gain $(\mathrm{G})$ as the ratio $\mathrm{G}=\left(\mathrm{V}^{*} \neq\right)$. As the gain $(\mathrm{G})$ decreases, there is less potential surplus for the several small bidders to share in overcoming the large bidder, and it becomes more difficult for the small bidders to collaborate to achieve the optimal allocation.

Condition 2: The Own Effect. This effect is one that is coupled with the gain effect. It occurs when $\mathrm{j}$ is the large package bidder who demands $¥$, but $\mathrm{j}$ is also one of the small package bidders 
included in $\mathrm{V}^{*}$. To achieve the optimal allocation $\mathrm{j}$ must forego his large package to be included in the optimal allocation of smaller winning packages. Efficiency may be hurt because $\mathrm{j}$ may not collaborate in his role as a small package bidder for two reasons: first, he feels he is in a stronger negotiating position (since he values the large package) and may demand more of the surplus than other small package bidders; or second, he may think that displacing himself in the large package, even if it is apparently profitable, may create unpredictable dynamics in the subsequent bidding.

Figures 6 and 7 show the various boundary environments we tested using ten licenses (A through J). Actual parameters for these experiments also can be found at the site,

http://linus.econlab.arizona.edu/FCC Parameters In Figure 6 we see that bidder 6 had the large package and was either in the winning set or not with package F,G (own effect). The Gain was either lowest at $1.23(430 / 350)$ or highest at $1.43(430 / 301)$.

In Figure 7 we divided the licenses into two groups with various own and gain effects. For licenses A-E we had several bidders with values for the entire license group from A to E, while some of them had values for packages that were part of the optimal allocation set. In the second group of licenses F-J, each bidder has values for single licenses except bidder 5 who has value for the entire group of licenses F-J. We divide these two groups into Cases $2 \mathrm{a}$ and $2 \mathrm{~b}$ respectively.

\subsubsection{Boundary Case Results}

Table 30 shows the raw efficiencies and design parameters for all the boundary experiments we conducted.

Result 16: For each value environment, decreased gain reduced efficiency and the presence of the own effect also reduced efficiency.

While there seems to be a gain and own effect, we notice that in Case $2 a$, in which the gain is at the "low" level of Case 1 and there is an own effect, efficiencies are relatively much higher and similar across auction forms. This suggests that the impact of the gain and own effects are interrelated with the constellations of packages being sought. Furthermore, though efficiencies are low for the CMA in Case 1, they are higher than for the SMA, while in Case 2b 
the SMA outperforms the CMA. Thus, the value environment and rules of the institutions tested interacted to determine efficiency. To choose between the versions of the SMA and CMA tested, one might need more a priori knowledge of the environment, than it is possible to obtain.

\subsubsection{Implementation and Strategic Issues}

One design parameter for which there was no implementation specification provided with the Charles River and Associates' CMA rules, was the amount of initial eligibility to be awarded each participant. Setting initial eligibility just equal to the number of licenses available (10 in our experiment) can severely restrict the number of packages that one can simultaneously submit. This would hamper the ability of bidders to find fits and the optimization algorithm to do its work. After some preliminary trials, we set the initial eligibility at 30 points, which allowed the bidders the possibility to express an opening interest in all packages that were of value to them.

One strategic ploy we observed in several of the auctions was manifested by the following bidding tactic. Bidder $\mathrm{i}$ would place a winning bid on a large valuable package, say $\mathrm{ABCD}$, and also place a high bid on the subset package $\mathrm{AB}$ for which he had little value, but which was being pursued by another bidder $\mathrm{j}$. In the next round, to remain eligible bidder $\mathrm{j}$ would have been forced to bid above the AB bid placed in the previous period by bidder $\mathrm{i}$, though i was free to immediately, and unannounced, remove that bid. ${ }^{29}$ This lack of required commitment on the previous round in non-winning bids can provide poor information for competitive bidders, affecting eligibility constraints and auction efficiency.

\section{Summary}

The Simultaneous Multi-round Auction (SMA) employed by the Federal Communications Commission may contain several rules that unnecessarily complicate the task

of assessing the comparative performance characteristics of the SMA and the Charles River and Associates Combinatorial Multi-round Auction (CMA). Our study uses economic experiments to examine the effect that two distinct yet interrelated rules have on the performance of the SMA. The first rule assigns unequal eligibility points to different licenses, and the second rule controls the amount of flexibility that the bidder maintains during the course of the auction for a given level of bidding activity. These two rules were collectively referred to as the Eligibility Rules. 
The two-part structure of the current rule suggested the evaluation of the alternatives in the following 2 x 2 design: Equal vs. Unequal Eligibility points cross Flexible vs. Inflexible Eligibility.

\section{Alternative Eligibility Rules}

\begin{tabular}{|c|c|c|}
\hline & Nonflexible & Flexible \\
\hline Equal & Baseline & Flexible Eligibility \\
\hline Unequal & License Differentiation & Flexible Eligibility \& \\
& & License Differentiation \\
\hline
\end{tabular}

Experiments were conducted to examine the performance properties of all four Eligibility Rules. Because the results of an experiment may be sensitive to the bidder valuation environment within which it is conducted, experiments were run under four different bidder valuation environments: (1) additive; (2) low superadditivity; (3) medium superadditivity; and (4) high superadditivity. A variety of measures were used to evaluate auction performance, the most important of which were assignment efficiency, revenue, and auction length.

The results of the experiments indicate that there is no statistically significant difference in the performance properties of the four alternative eligibility rules when the value a bidder places on any set of licenses is "additive" (equal to the sum of the values of the individual licenses that comprise the set). Moreover, in the additive valuation environment, none of the Eligibility Rules have a statistically significant effect on auction revenue or on the level of bidder losses that occurred. Under all four Eligibility Rules, bidder losses were either zero or negligible.

Analyses were also conducted to examine whether there are statistically significant differences in the performance properties of the four different Eligibility Rules when the value any bidder places on a set of licenses is superadditive (greater than the sum of the values of the individual licenses that comprise the set). They reveal that the increased flexibility in eligibility has a positive effect on assignment efficiency. Moreover, the analyses reveal that assignment efficiency is not affected by whether licenses are assigned unequal or equal points, and is also independent of the level of superadditivity in the valuation that bidders place on a set of licenses.

The analyses additionally reveal that flexible eligibility and the assignment of equal eligibility points across all licenses have significant positive effects on auction revenue. We also 
find that an increase in the average size of the packages of licenses that generate superadditive value also increases auction revenue. While the adoption of flexible eligibility enhances efficiency by $3 \%$, the analyses also indicate that such a rule increases the average auction length by $20 \%$. This last result offsets to an unknown degree the value of the increase in efficiency. The Charles River and Associates' CMA yielded significantly higher efficiency, and significant lower revenue in all super additive environments, than did the SMA procedure. The higher revenue in the SMA was a consequence of losses incurred by bidders in failed (package) license aggregations. The better performance of the CMA, however, was attained at a transactions' cost of requiring three times as many auction rounds to complete the auctions. In many, but not all of the experiments, additional rounds did not change allocations or revenue, but this was not known by the participants who were ardently monitoring the process and seeking (without computational 'cobbling' assistance) from the mechanism their best alternatives in the form of collaborative package combinations.

One of the primary objectives of auction design should be to simplify, and reduce the cost of the bidding process for the participants. This may require mechanism computational support in the form of optimization algorithms when environments are superadditive. The auction should incentivize bidders to devote their resources to determining the value to themselves of the various individual and packages of licenses. The auction should provide a simple and clear way for bidders to declare their value structure, no matter how complicated, without fear of others free-riding on their investment in estimating or developing value. ${ }^{30}$ The auction should not obligate bidders to expend an inordinate amount of resources on consultant and management time trying to figure out how to bid strategically in order to realize their potential value.

It has been argued abstractly that combinatorial bidding is computationally infeasible because in a large FCC auction the number of alternative combinations of elements exceeds the number of particles in the universe (MacAfee and McMillan). But this is quite irrelevant, since bidders, like grocery shoppers, are only interested in a small portion of the commodity space. It has also been noted correctly that the cost of preparing a bid limits the number of combinations that a bidder will want to consider (Milgrom, 2000), even if he has potential interest in many. Wherever bidding environments are superadditive there is no excuse for mechanism designers to ignore the benefits of $21^{\text {st }}$ century technology in lowering the cost and complexity for the participants in conducting the auction. Both efficiency of allocation and transparency to the 
participants require that the auction be organized in one of the several 'smart' computer assisted forms that are now practical.

The FCC experience in auction design, learning from its application, redesigning in the light of emergent design flaws, more learning in application etc., provides important lessons in parallelism between field and laboratory for experimentalists, theorists and economist generally, who have an interest in economic design:

(1) Many of the issues and attendant learning in laboratory experiments about strategic ("gaming") behavior - jump bidding, new forms of gaming induced by rules designed to limit strategic behavior, increased transactions cost and/or auction length induced by controls for gaming, collusive attempts when bidders cannot bid anonymously - subsequently emerged and were relearned in the FCC auction through similar parallel sequential experiences. (McCabe, Rassenti and Smith, 1988/1991).

(2) Besides providing new evidence on parallelism between the two study environments, FCC experience argues strongly for more intensive laboratory test bedding of proposed new FCC and other complex auction mechanisms. Elementary errors and their correction in mechanism design should be made in the laboratory, not in the field where the cost is very large and borne by others besides the designers and their founders. This argument is further supported by laboratory studies and field applications of market mechanisms for trading emission rights (Isikida, et. al.) and for electric power trading (Rassenti, Smith and Wilson, 2001).

(3) All of the SMA and CMA experiments reported here are based on induced private value environments, as were most of the background English auction experiments summarized in Part 1. The issues in (1) and (2) are therefore not dependent on common value elements in the environment, nor, as indicated in the discussion of Part 1 , does there appear to be any sound theoretical or empirical justification for continuing to think of such environments as being central or even of marginal concern in auction design or in the interpretation of field evidence. 


\section{Footnotes}

1. The so-called "English" auction was imported to the British Islands by the Romans. This is indicated by word origins. Thus, the word auction is derived from the Latin root auctio meaning “an increasing”(procedure). More revealing, the Oxford English Dictionary notes that an old English word, fallen into disuse, is subhasta, which is still used in Spain to refer to an auction or auction house. This word is compounded from the Latin words sub hasta meaning (a sale) "under the spear" which refers to the practice of the auctioneers who followed the Roman army to auction the spoils of war so that the soldiers would not have to be paid in kind.

2. Some auctions reduce transaction cost by defining off-floor bidding procedures: stamp (and some fish) auctions permit "book bids" by buyers not present at the auction, and Southby's, for example, allows buyers to monitor by telephone and submit oral bids to the auctioneer for paintings and collectibles. Book bids are of theoretical interest because they long ago made it transparent to practicing auction houses that the second price sealed bid auction is isomorphic to the English auction. Hence, the rule that if there is one book bid, the auctioneer calls for a bid from the floor. Given a bid from the floor the auctioneer advances the bid, for the account of the book bidder by the standard increment, $\Delta$. If this bid is advanced from the floor, the auctioneer again advances the bid by $\Delta$ for the book bidder, and so on, until either the book bidder is stopped out by a floor bidder, or bidding from the floor ceases, in which case the item is knocked down to the book bidder at a price equal to the last standing floor bid plus $\Delta$. If there are two (or more) book bids, then the auctioneer starts the bidding at the second highest book bid plus $\Delta$. If there are no counter bids from the floor, the award is to the highest book bid at $\Delta$ over the second book bid.

3. In McCabe, Rassenti and Smith (1990) a ninth mechanism - the multiple unit uniform price Dutch auction -is modelled theoretically, and tested.

4. An exception is in several market-like public good mechanisms that appeared in the period 1977-1984 using the Groves-Ledyard and other mechanisms. (See Smith, 1991, Part III, pp. 375-506; also Chapter 2 by Ledyard in Kagel and Roth, 1995.)

5. All the indicated value information is private for each agent. The mechanism allows bids of the form: "Accept no more than $p$ of the following $q$ packages;" "don't spend more than $\$ M$; accept package A only if B is accepted." Any logical constraints linear in the $(0,1)$ variables are acceptable. The experiments did not utilize these bid options.

6. For auctions in which the number of single and package items is not so large as to be computationally infeasible, the incentive compatible Vickrey auction is an obvious candidate. Bid preparation costs are a disadvantage, but large savings would be obtained: it would eliminate the multiple round format entirely, as well as the need to hire auction consultants.

7. For example, if the scalar is 3 in a given round, and the bidder submits a bid on a license that has 10 eligibility points associated with it, then in the next round the bidder has the flexibility 
to submit bids on licenses that total up to 30 points, or the current round's maximum eligibility (a predetermined number), whichever is less.

8. We imposed what the FCC refers to as "click-box" bidding. This form of bidding allows the bidder to only increase its bid in integer multiples of the identified increment. Thus, if the increment amount were $10 \%$ for a particular license, any bid submitted for that license was restricted to be Standing Bid times $(1+.10 \pi)$, where $\pi$ is a positive integer greater than or equal to 1 .

9. Because a standing bid on a license may be withdrawn multiple times, the highest bid after a withdrawal need not be the final bid on a license.

10. Also, with agreement from the FCC, we combined the bidding and withdrawal phases of the auction. While this speeds up the auction, it does create an uncertainty on the part of the withdrawing party who cannot signal the availability of a license before bidding begins.

11. The decision not to include such a rule was approved by the FCC prior to conducting the experiments.

12. The tapered SMR auction uses stages to gradually restrict the number of licenses on which a participant can bid. Let $\mathrm{j}=1,2 \ldots \mathrm{M}$ denote the stage of the auction. For each stage there is a number $\omega_{\mathrm{j}} \geq 1$ such that $\omega_{1}>\omega_{2}>\ldots .>\omega_{\mathrm{M}}$. We did not use any stages in order to isolate the effect of the increased flexibility in tapered auctions.

13. A value function $\mathrm{V}(\cdot)$ is said to be additive if for any subset of licenses $\mathrm{L}$, the total value of the subset is the sum of individual values $V_{j}$ for each license: $V(L)=\Sigma_{j \in L} V_{j}$.

14. We ignore the subadditive case because of limited resources to investigate it. Resources permitting, at a later date we will examine the case in which bidders are budget constrained which has a similar effect.

15. We correlated these values with the number of eligibility points associated with each license.

16. This structure is somewhat similar to the econometric model developed by Ausubel et al. (1997).

17. A competitive equilibrium in this environment is a set of license prices such that there would be only one demander per license at those prices and this would be the efficient allocation.

18. The algorithm used to select values included a probability function $\mathrm{p}_{\mathrm{ij}}$ that determined whether license $\mathrm{j}$ would be in package $\mathrm{i}$ for a bidder. When this probability function is increased, average package size is increased and package overlap across subjects is more likely.

19. Any set of licenses that generated synergy values did not contain elements from both sets Фand $\Psi$. 
20. The non-existence of a set of competitive equilibrium prices in some superadditive environments makes it impossible to pool the additive and superadditive data, the former of which always has a set of competitive equilibrium prices.

21. It was necessary for us to have cases with different numbers of bidders due to the possibility of bankruptcy eliminating some participants.

22. Recall that the largest price increment in our SMA experiments was $15 \%$ above the standing bid.

23. A package bid is defined as a bid for two or more specified licenses in which all licenses in the package must be accepted at the package bid, or none should be accepted.

24. The reason for retaining old bids that do not meet the minimum bid requirement is to facilitate possible package combinations in later rounds, even though the current bid alone is not in the winning set.

25. Even though new bids are submitted it does not mean that revenue has increased. This occurs because an acceptable bid must be better than the best bids in the system that the new bid replaces, not the winning bids.

26. Any set of licenses that generated synergy values did not contain elements from both sets Фand $\Psi$.

27. In this report, we do not use the experiments from the treatments in Part 2 in which licenses had the same number of eligibility points.

28. We could measure efficiency to include external reductions of working capital.

29. For example suppose $i$ has a value of 800 for $A B C D$ while $j$ has a value of 500 for $A B$ and $k$ has a value of 500 for $\mathrm{CD}$. Suppose there are other items that $\mathrm{j}$ and $\mathrm{k}$ have value for, but in the optimal allocation they should combine to win $A B$ and $C D$. Suppose $j$ and $k$ currently hold the winning bids of 50 for $\mathrm{AB}$ and $\mathrm{CD}$ respectively. Employing the above technique, $\mathrm{i}$ can bid 600 for $\mathrm{ABCD}$ and 500 for $\mathrm{AB}$. Bidder $\mathrm{j}$ will be turned away to spend his eligibility on other more affordable licenses, while i simply removes his $\mathrm{AB}$ bid immediately, having denied $\mathrm{k}$ an opportunity to collaborate with $\mathrm{j}$.

30. To both minimize strategic behavior and encourage the estimation and development of private value, the information feedback to the bidders must be limited to the minimum required for an individual bidder to know if his/her bid is a potential or actual winner. One simple example is provided by the English clock auction . An active bidder is informed only that there remains at least one other bidder on any item, the stopping rule being that as soon as the penultimate bidder drops out, the auction for that item is closed. Every bidder remains anonymous. 


\section{Appendix A:}

Table A1 Experimental Design

\begin{tabular}{|c|c|c|c|}
\hline Treatment & Experiment & Date & Environments $(\Phi, \Psi)$ \\
\hline SMA & 1 & $4 / 16 / 99$ & Additive, Medium \\
\hline SMA & 1 & $4 / 16 / 99$ & Low, High \\
\hline SMA & 1 & $4 / 16 / 99$ & High, Additive \\
\hline SMA & 2 & $4 / 21 / 99$ & Additive, Medium \\
\hline SMA & 2 & $4 / 21 / 99$ & Low, High \\
\hline SMA & 2 & $4 / 21 / 99$ & High, Additive \\
\hline SMA & 3 & $4 / 27 / 99$ & Low, High \\
\hline SMA & 4 & $5 / 3 / 99$ & Medium, Low \\
\hline SMA & 4 & $5 / 3 / 99$ & Medium, Low \\
\hline SMA & 4 & $5 / 3 / 99$ & Low, High \\
\hline SMA & 4 & $5 / 3 / 99$ & Medium, Low \\
\hline SMA & 5 & $5 / 4 / 99$ & Medium, Low \\
\hline SMA & 5 & $5 / 4 / 99$ & Low, High \\
\hline SMA & 5 & $5 / 4 / 99$ & High, Additive \\
\hline SMA & 6 & $5 / 7 / 99$ & Additive, Medium \\
\hline SMA & 6 & $5 / 7 / 99$ & High, Additive \\
\hline SMA & 6 & $5 / 7 / 99$ & Low, High \\
\hline SMA & 7 & $8 / 3 / 99$ & Low, High \\
\hline SMA & 8 & $4 / 27 / 99$ & Medium, Low \\
\hline SMA & 9 & $4 / 29 / 99$ & Additive, Medium \\
\hline SMA & 9 & $4 / 29 / 99$ & High, Additive \\
\hline SMA & 9 & $4 / 29 / 99$ & Additive, High \\
\hline SMA & 10 & $4 / 30 / 99$ & Additive, Medium \\
\hline SMA & 10 & $4 / 30 / 99$ & Low, High \\
\hline SMA & 10 & $4 / 30 / 99$ & High, Additive \\
\hline SMA & 11 & $5 / 3 / 99$ & Additive, High \\
\hline SMA & 11 & $5 / 3 / 99$ & Medium, Low \\
\hline SMA & 11 & $5 / 3 / 99$ & Medium, Low \\
\hline SMA & 11 & $5 / 3 / 99$ & High, Additive \\
\hline SMA & 12 & $5 / 4 / 99$ & Additive, Medium \\
\hline SMA & 12 & $5 / 4 / 99$ & Medium, Low \\
\hline SMA & 12 & $5 / 4 / 99$ & Medium, Low \\
\hline SMA & 12 & $5 / 4 / 99$ & High, Additive \\
\hline SMA & 13 & $5 / 7 / 99$ & High, Additive \\
\hline SMA & 13 & $5 / 7 / 99$ & Medium, Low \\
\hline SMA & 14 & $8 / 3 / 99$ & Low, High \\
\hline Combinatorial & 1 & $10 / 2 / 99$ & Medium, Low \\
\hline Combinatorial & 2 & $11 / 2 / 99$ & Medium, Low \\
\hline Combinatorial & 2 & $11 / 2 / 99$ & Medium, Low \\
\hline Combinatorial & 3 & $11 / 5 / 99$ & High, Additive \\
\hline Combinatorial & 3 & $11 / 5 / 99$ & Additive, High \\
\hline Combinatorial & 4 & $11 / 8 / 99$ & Low, High \\
\hline Combinatorial & 4 & $11 / 8 / 99$ & Additive, Medium \\
\hline Combinatorial & 5 & $11 / 9 / 99$ & Medium, Low \\
\hline Combinatorial & 5 & $11 / 9 / 99$ & Additive, High \\
\hline Combinatorial & 6 & $11 / 10 / 99$ & Low, High \\
\hline Combinatorial & 6 & $11 / 10 / 99$ & Additive, Medium \\
\hline Combinatorial & 7 & $11 / 11 / 99$ & Low, High \\
\hline Combinatorial & 7 & $11 / 11 / 99$ & High, Additive \\
\hline Combinatorial & 7 & $11 / 11 / 99$ & Low, High \\
\hline Combinatorial & 8 & $11 / 12 / 99$ & High, Additive \\
\hline Combinatorial & 8 & $11 / 12 / 99$ & Medium, Low \\
\hline Combinatorial & 9 & $11 / 15 / 99$ & Low, High \\
\hline Combinatorial & 9 & $11 / 15 / 99$ & Additive, High \\
\hline Combinatorial & 10 & $11 / 16 / 99$ & Medium, Low \\
\hline Combinatorial & 10 & $11 / 16 / 99$ & Additive, Medium \\
\hline Combinatorial & 11 & $11 / 19 / 99$ & Medium, Low \\
\hline Combinatorial & 11 & $11 / 19 / 99$ & Additive, High \\
\hline Combinatorial & 12 & $11 / 22 / 99$ & High, Additive \\
\hline Combinatorial & 13 & $11 / 23 / 99$ & High, Additive \\
\hline
\end{tabular}


Table A2 Experimental Design

\begin{tabular}{|c|c|c|c|}
\hline Treatment & Experiment & Date & Environments $(\Phi, \Psi)$ \\
\hline Baseline & 1 & $4 / 16 / 99$ & Additive, High \\
\hline Baseline & 1 & $4 / 16 / 99$ & Low, Additive \\
\hline Baseline & 1 & $4 / 16 / 99$ & High, Medium \\
\hline Baseline & 2 & $4 / 21 / 99$ & Additive, High \\
\hline Baseline & 2 & $4 / 21 / 99$ & Low, Additive \\
\hline Baseline & 2 & $4 / 21 / 99$ & High, Medium \\
\hline Baseline & 3 & $4 / 27 / 99$ & Low, Additive \\
\hline Baseline & 4 & $5 / 3 / 99$ & Medium, Low \\
\hline Baseline & 4 & $5 / 3 / 99$ & Medium, Low \\
\hline Baseline & 4 & $5 / 3 / 99$ & Low, Additive \\
\hline Baseline & 4 & $5 / 3 / 99$ & Medium, Low \\
\hline Baseline & 5 & $5 / 4 / 99$ & Medium, Low \\
\hline Baseline & 5 & $5 / 4 / 99$ & Low, Additive \\
\hline Baseline & 5 & $5 / 4 / 99$ & High, Medium \\
\hline Baseline & 6 & $5 / 7 / 99$ & Additive, High \\
\hline Baseline & 6 & $5 / 7 / 99$ & High, Medium \\
\hline Baseline & 6 & $5 / 7 / 99$ & Low, Additive \\
\hline Baseline & 7 & $8 / 3 / 99$ & Low, Additive \\
\hline Flexible & 1 & $4 / 27 / 99$ & Medium, Low \\
\hline Flexible & 2 & $4 / 29 / 99$ & Additive, High \\
\hline Flexible & 2 & $4 / 29 / 99$ & High, Medium \\
\hline Flexible & 2 & $4 / 29 / 99$ & Additive, High \\
\hline Flexible & 3 & $4 / 30 / 99$ & Additive, High \\
\hline Flexible & 3 & $4 / 30 / 99$ & Low, Additive \\
\hline Flexible & 3 & $4 / 30 / 99$ & High, Medium \\
\hline Flexible & 4 & $5 / 3 / 99$ & Additive, High \\
\hline Flexible & 4 & $5 / 3 / 99$ & Medium, Low \\
\hline Flexible & 4 & $5 / 3 / 99$ & Medium, Low \\
\hline Flexible & 4 & $5 / 3 / 99$ & High, Medium \\
\hline Flexible & 5 & $5 / 4 / 99$ & Additive, High \\
\hline Flexible & 5 & $5 / 4 / 99$ & Medium, Low \\
\hline Flexible & 5 & $5 / 4 / 99$ & Medium, Low \\
\hline Flexible & 5 & $5 / 4 / 99$ & High, Medium \\
\hline Flexible & 6 & $5 / 7 / 99$ & High, Medium \\
\hline Flexible & 6 & $5 / 7 / 99$ & Medium, Low \\
\hline Flexible & 7 & $8 / 3 / 99$ & Low, Additive \\
\hline Unequal & 1 & 4/16/99 & Additive, High \\
\hline Unequal & 1 & $4 / 16 / 99$ & High, Medium \\
\hline Unequal & 1 & $4 / 16 / 99$ & Low, Additive \\
\hline Unequal & 2 & $4 / 21 / 99$ & Additive, High \\
\hline Unequal & 2 & $4 / 21 / 99$ & High, Medium \\
\hline Unequal & 2 & $4 / 21 / 99$ & Low, Additive \\
\hline Unequal & 2 & $4 / 21 / 99$ & Medium, Low \\
\hline Unequal & 3 & $4 / 28 / 99$ & Medium, Low \\
\hline Unequal & 3 & $4 / 28 / 99$ & Additive, High \\
\hline Unequal & 3 & $4 / 28 / 99$ & High, Medium \\
\hline Unequal & 3 & $4 / 28 / 99$ & Medium, Low \\
\hline Unequal & 4 & $4 / 30 / 99$ & Medium, Low \\
\hline Unequal & 4 & $4 / 30 / 99$ & Additive, High \\
\hline Unequal & 4 & $4 / 30 / 99$ & High, Medium \\
\hline
\end{tabular}




\begin{tabular}{|l|l|l|l|}
\hline Unequal & 4 & $4 / 30 / 99$ & Medium, Low \\
\hline Unequal & 4 & $5 / 3 / 99$ & Low, Additive \\
\hline Unequal & 4 & $5 / 3 / 99$ & High, Medium \\
\hline Unequal, Flexible & 1 & $4 / 20 / 99$ & Additive, High \\
\hline Unequal, Flexible & 1 & $4 / 20 / 99$ & Low, Additive \\
\hline Unequal, Flexible & 1 & $4 / 20 / 99$ & High, Medium \\
\hline Unequal, Flexible & 2 & $4 / 23 / 99$ & Additive, High \\
\hline Unequal, Flexible & 2 & $4 / 23 / 99$ & Low, Additive \\
\hline Unequal, Flexible & 2 & $4 / 23 / 99$ & Medium, Low \\
\hline Unequal, Flexible & 2 & $4 / 23 / 99$ & High, Medium \\
\hline Unequal, Flexible & 3 & $4 / 26 / 99$ & Additive Medium \\
\hline Unequal, Flexible & 3 & $4 / 26 / 99$ & Additive, High \\
\hline Unequal, Flexible & 3 & $4 / 26 / 99$ & High, Medium \\
\hline Unequal, Flexible & 4 & $4 / 28 / 99$ & Medium, Low \\
\hline Unequal, Flexible & 5 & $4 / 29 / 99$ & Medium, Low \\
\hline Unequal, Flexible & 5 & $4 / 29 / 99$ & High, Medium \\
\hline Unequal, Flexible & 5 & $4 / 29 / 99$ & Medium, Low \\
\hline Unequal, Flexible & 6 & $5 / 7 / 99$ & Additive, High \\
\hline Unequal, Flexible & 6 & $5 / 7 / 99$ & Low, Additive \\
\hline Unequal, Flexible & 7 & $8 / 3 / 99$ & Low, Additive \\
\hline & & & \\
\hline
\end{tabular}




\section{References}

Ausubel, L.M., Cramton, P., McAfee, R.P., McMillan, J., 1997. Synergies in wireless telephony: evidence from the MTA auction. Journal of Economics and Management Strategy 6(3), 497-527.

Banks, J., Ledyard, J., Porter, D., 1989. Allocating uncertain and unresponsive resources: an experimental approach. Rand Journal of Economics 20, 1-25.

Berg, J., Dickhaut, J., McCabe, K., 1986. Risk preference instability across institutions: a dilemma. University of Minnesota, May.

Bossarts, P., Fine, L., Ledyard, J., 2000. Inducing liquidity in thin financial markets through combined-value trading mechanisms. California Institute of Technology.

Brewer, P., Plott, C., 1996. A binary conflict ascending price (BICAP) mechanism for the decentralized allocation of the right to use railroad tracks. International Journal of Industrial Organization 14, 857-886.

Bykowsky, M., Cull, R., 1998. Bidder non-neutrality and radio spectrum auctions. mimeo, NTIA, Washington, D.C.

Bykowsky M., Cull, R., Ledyard, J., 2000. Mutually destructive bidding: the Federal Communications Commission auction design problem. Journal of Regulatory Economics 17(3), 205-228.

Cassady, R., 1967. Auctions and Auctioneering. Berkeley: University of California Press.

Charles River and Associates Inc. and Market Design Inc., 1998. Report 1A: Auction design enhancements for non-combinatorial auctions. Charles River and Associates No. 135100 .

Charles River and Associates Inc. and Market Design Inc., 1998. Report 1B: Package bidding for spectrum licenses. Charles River and Associates No. 1351-00.

Charles River and Associates Inc. and Market Design Inc., 1998. Report 2: Simultaneous ascending auctions with package bidding. Charles River and Associates No. 1351-00, March 1998.

Chew, S.H., Nishimura, N., 1999. Revenue non-equivalence between the English and the second price auctions: experimental evidence. Hong Kong University of Science and Technology.

Coppinger, V., Smith, V., Titus, J., 1980. Incentives and behavior in English Dutch and sealedbid auction. Economic Inquiry 18, 1-22.

Cox, J., Roberson, B., Smith, V., 1982. Theory and behavior of single object auctions, In: V. Smith (Ed.), Research in Experimental Economics, Vol. 2. Greenwich, CT: JAI Press.

Cox, J., Isaac, M., Smith, V., 1983. OCS leasing and auction. Supreme Court Economic Review.

Cox J., Smith V., 1992. Endogenous entry and exit in common value auction.” Mimeographed, Economic Science Laboratory, University of Arizona.

Cox, J., Grether, D., 1996. The preference reversal phenomenon: response mode, markets, and incentives. Economic Theory 7(3), 381-405.

Garvin, S., Kagel, J., 1994. Learning in common value auctions. Journal of Economic Behavior and Organization 25, 351-372.

Isikida, T., Ledyard, J., Olson, M., Porter, D., 2000. Experimental testbedding of a pollution trading system: Southern California RECLAIM Emissions Market, In: R. Isaac (Ed.), Research in Experimental Economics. Greenwich, CT: JAI Press.

Jackson, M., 1999. The non-existence of equilibrium in auctions with two dimensional types. Division of Humanities and Social Sciences, 228-77, Caltech, Pasadena, CA. 
Kagel, J.H., Levin D., 1986. The winner's curse and public information in common value auctions. American Economic Review 76, 894-920.

Kagel, J., Roth, A., 1995. Handbook of Experimental Economics. Princeton, NJ: Princeton University Press.

Klemperer, P., 1998. Auctions with almost common values: the 'Wallet Game' and its applications. European Economic Review.

Ledyard, J., Porter, D., Rangel, A., 1994. Using computerized exchange system to solve a project management problem. Journal of Organizational Computing.

Ledyard, J., Porter, D., Rangel, A., 1997. Experiment testing multiobject allocation mechanism. Journal of Economics and Management Strategy 6, 639-675.

Ledyard, J., Olson, M., Porter, D., Swanson, J., Torma, D., 2000. The Sears Logistics Auction. Informs, forthcoming.

Lucking-Reiley, D., 1999. Auctions on the internet: what's being auctioned, and how?" Department of Economics, Vanderbilt University.

McAfee, R.P., McMillan, J., 1996. Analyzing the airways auction. Journal of Economic Perspectives 10, 159-175.

McCabe, K., Rassenti, S., Smith, V., 1989. Designing 'smart' computer assisted markets. European Journal of Political Economy 5, 259-83.

McCabe, K., Rassenti, S., Smith, V., 1988. Testing Vickrey's and other simultaneous multiple unit versions of the English auction, revised 1989. In: R. M. Isaac (Ed). Research in Experimental Economics, Vol. 4, 1991. Greenwich, CT: JAI Press.

McCabe, K., Rassenti, S., Smith, V., 1990. Auction institutional design: theory and behavior of simultaneous multiple unit generalizations of the Dutch and English auctions. American Economic Review 80, 1276-83.

McMillan, J., 1994. Selling spectrum rights. Journal of Economic Perspectives.

Milgrom, P., 2000. Putting auction theory to work: the simultaneous ascending auction. Journal of Political Economy.

Milgrom, P., Weber, R., 1982. A theory of auctions and competitive bidding. Econometrica 50, 1485-1527.

Olson, M., Rassenti, S., Smith, V., Rigdon, M., 2000. Market design and motivated human trading behavior in electricity markets. IIE Transactions on Operations Engineering.

Porter, D., Vragov, R., 2000. An experimental examination of demand reduction in multi-unit versions of the uniform-price, Vickrey, and English auctions. University of Arizona.

Rassenti, S., 1981. O-1 decision problems with multiple resource constraints: algorithms and applications. Unpublished Ph.D. Thesis, University of Arizona.

Rassenti, S., Smith, V., Bulfin, R., 1982. A combinatorial auction mechanism for airport time slot allocation. Bell Journal of Economics, 13, 402-417.

Rassenti, S., Smith, V., 1986. Electric utility deregulation, In: Pricing Electric, Gas and Telecommunication Services, The institute for the Study of regulation, December.

Rassenti, S., Smith, V., Wilson, B., 2001. Using experiments to inform the privatization/deregulation movement in electricity. To appear Cato Journal.

Salant, D., 1995. Up in the air: GTE's experience in the MTA auctions for PCS licenses. Mimeo, Charles Rivers and Associates.

Smith, V., 1991. Papers in Experimental Economics. Cambridge: Cambridge University Press. Vickrey, W., 1961. Counterspeculation, auctions and competitive sealed tenders. Journal of Finance 16, 8-37. 
Vickrey, W., 1976. Auction, markets, and optimal allocations. In: Y. Amihud (Ed.), Bidding and Auctioning for Procurement and Allocation. New York: New York University Press. 
Table 1: License Eligibility Point Assignments

\begin{tabular}{|c|c|c|c|c|c|c|c|c|c|c|}
\hline License & A & B & C & D & E & F & G & H & I & J \\
\hline Points & 1 & 2 & 3 & 4 & 5 & 1 & 2 & 3 & 4 & 5 \\
\hline
\end{tabular}


Table 2: 2 x 2 Experimental Treatments

\begin{tabular}{|c|c|c|}
\hline & Nonflexible & Flexible \\
\hline Equal & Baseline & Flexible Eligibility \\
\hline Unequal & Point Differentiation & Flexible Eligibility \& \\
& & Point Differentiation \\
\hline
\end{tabular}


Table 3: Parameter Values

\begin{tabular}{|c|c|c|c|c|}
\hline Environment & $\lambda$ & $\beta$ & $\Delta$ & $\alpha$ \\
\hline High & 175 & 1 & 230 & 2.05 \\
\hline Medium & 150 & 1 & 229 & 1.65 \\
\hline Low & 78 & .65 & 120 & 1.65 \\
\hline
\end{tabular}


Table 4: Mean (Median) Efficiency by Treatment: Additive Environment

\begin{tabular}{|c|c|c|}
\hline & Nonflexible & Flexible \\
\hline Equal & $96.4(100)$ & $96.9(100)$ \\
\hline Unequal & $93.6(93.8)$ & $96.2(100)$ \\
\hline
\end{tabular}


Table 5: Count of $\mathbf{1 0 0 \%}$ Efficient Outcomes by Treatment: Additive Environment ( $n=$ number of total experiments)

\begin{tabular}{|c|c|c|}
\hline & Nonflexible & Flexible \\
\hline Equal & $6(n=10)$ & $5(n=7)$ \\
\hline Unequal & $3(n=7)$ & $6(n=9)$ \\
\hline
\end{tabular}


Table 6: Linear Model Parameter Estimates for Efficiency: Additive Environment

\begin{tabular}{|c|c|c|}
\hline Variable & Estimate & p-value \\
\hline Intercept & 99.2 & 0.00 \\
\hline Flexible & 1.02 & 0.35 \\
\hline Unequal & 1.05 & 0.34 \\
\hline Environment Pairing & 0.84 & 0.46 \\
\hline Number of Bidders & -1.43 & 0.31 \\
\hline
\end{tabular}


Table 7: Mean (Median) Efficiency by Treatment: Superadditive Environment

\begin{tabular}{|c|c|c|}
\hline & Nonflexible & Flexible \\
\hline Equal & $74.5(78.5)$ & $82.7(91.0)$ \\
\hline Unequal & $65.0(61.0)$ & $80.4(91.0)$ \\
\hline
\end{tabular}


Table 8: Count of $100 \%$ Efficient Outcomes by Treatment: Superadditive Environment

\begin{tabular}{|c|c|c|}
\hline & Nonflexible & Flexible \\
\hline Equal & $5(n=26)$ & $2(n=29)$ \\
\hline Unequal & $0(n=27)$ & $2(n=25)$ \\
\hline
\end{tabular}


Table 9: Linear Model Parameter Estimates for Efficiency: Superadditive Environment

\begin{tabular}{|c|c|c|}
\hline Variable & Estimate & p-value \\
\hline Intercept & 68.7 & 0.0 \\
\hline Flexible & 6.29 & 0.002 \\
\hline Unequal & 2.69 & 0.16 \\
\hline Level of Synergy (Medium) & -3.04 & 0.25 \\
\hline Level of Synergy (High) & -0.88 & 0.51 \\
\hline Number of Bidders & -1.69 & 0.48 \\
\hline Overlap & 3.31 & 0.05 \\
\hline CE Exists & -3.25 & 0.11 \\
\hline
\end{tabular}


Table 10: Normalized (Mean, Median) Revenue: Additive Environment

\begin{tabular}{|c|c|c|}
\hline Treatments & Nonflexible & Flexible \\
\hline Equal & $1.41,1.31$ & $1.45,1.45$ \\
\hline Unequal & $1.39,1.39$ & $1.31,1.29$ \\
\hline
\end{tabular}


Table 11: Linear Model Parameter Estimates for Normalized Revenue: Additive Environment

\begin{tabular}{|c|c|c|}
\hline Variable & Estimate & p-value \\
\hline Intercept & 1.46 & 0.0 \\
\hline Flexible & -0.01 & 0.84 \\
\hline Unequal & 0.04 & 0.21 \\
\hline Environment Pairing & -0.01 & 0.91 \\
\hline Number of Bidders & -0.03 & 0.44 \\
\hline
\end{tabular}


Table 12: Normalized Revenue (Mean, Median): Superadditive Environment

\begin{tabular}{|c|c|c|}
\hline Treatments & Nonflexible & Flexible \\
\hline Equal & $0.92,0.89$ & $0.98,0.91$ \\
\hline Unequal & $0.72,0.78$ & $0.84,0.86$ \\
\hline
\end{tabular}


Table 13: Linear Model Parameter Estimates for Normalized Revenue: Superadditive Environment

\begin{tabular}{|c|c|c|}
\hline Variable & Estimate & P-Value \\
\hline Intercept & 1.01 & 0.00 \\
\hline Flexible & 0.05 & 0.07 \\
\hline Unequal & 0.09 & 0.001 \\
\hline Level of Synergy (Medium) & -0.05 & 0.18 \\
\hline Level of Synergy (High) & 0.02 & 0.27 \\
\hline Number of Bidders & -0.04 & 0.26 \\
\hline Overlap & 0.0026 & 0.91 \\
\hline CE Exists & 0.01 & 0.68 \\
\hline
\end{tabular}


Table 14: Average Rounds per Auction and Eligibility Tapering

\begin{tabular}{|c|c|c|c|c|}
\hline Treatments $\backslash$ Environment & Additive & Low & Medium & High \\
\hline Baseline & 7.0 & 9.5 & 8.6 & 7.9 \\
\hline Flexible & 8.4 & 10.4 & 10.0 & 9.3 \\
\hline Unequal & 6.7 & 7.3 & 7.4 & 6.8 \\
\hline Unequal $\wedge$ Flexible & 9.3 & 10.2 & 9.4 & 10.1 \\
\hline
\end{tabular}


Table 15: Poisson Model Parameter Estimates for Bidding Rounds

\begin{tabular}{|c|c|c|}
\hline Variable & Estimates & t-value \\
\hline Intercept & 2.10 & 25.2 \\
\hline Flexible & 0.12 & 4.15 \\
\hline Unequal & 0.02 & 0.87 \\
\hline Environment (Low) & 0.09 & 2.19 \\
\hline Environment (Medium) & 0.01 & 0.52 \\
\hline Environment (High) & -0.01 & -0.29 \\
\hline CE Exists & -0.04 & -1.16 \\
\hline Overlap & 0.01 & 0.43 \\
\hline
\end{tabular}


Table 16: Per Auction Losses: Additive Environment

\begin{tabular}{|c|c|c|}
\hline & Nonflexible & Flexible \\
\hline Equal & $\$ 0.15$ & $\$ 0.00$ \\
\hline Unequal & $\$ 0.01$ & $\$ 0.00$ \\
\hline
\end{tabular}


Table 17: Per Auction Losses: Superadditive Environments

Low

\begin{tabular}{|c|c|c|}
\hline & Nonflexible & Flexible \\
\hline Equal & $\$ 15.02$ & $\$ 11.31$ \\
\hline Unequal & $\$ 6.10$ & $\$ 3.64$ \\
\hline
\end{tabular}

Medium

\begin{tabular}{|c|c|c|}
\hline & Nonflexible & Flexible \\
\hline Equal & $\$ 5.79$ & $\$ 5.09$ \\
\hline Unequal & $\$ 6.16$ & $\$ 7.04$ \\
\hline
\end{tabular}

High

\begin{tabular}{|c|c|c|}
\hline & Nonflexible & Flexible \\
\hline Equal & $\$ 12.93$ & $\$ 21.39$ \\
\hline Unequal & $\$ 8.76$ & $\$ 10.15$ \\
\hline
\end{tabular}


Table 18: Linear Model Parameter Estimates for Losses per Bidder: Superadditive Environment

\begin{tabular}{|c|c|c|}
\hline Variable & Estimates & p-value \\
\hline Intercept & -278.4 & 0.001 \\
\hline Flexible & -0.56 & 0.97 \\
\hline Unequal & -35.78 & 0.097 \\
\hline Environment (Medium) & 42.48 & 0.141 \\
\hline Environment (High) & -25.9 & 0.08 \\
\hline Number of Bidders & 35.4 & 0.18 \\
\hline CE exists & -11.9 & 0.59 \\
\hline Overlap & 21.5 & 0.25 \\
\hline
\end{tabular}


Table 19: Per Auction Bankruptcies:

All Auctions

\begin{tabular}{|c|c|c|}
\hline & Nonflexible & Flexible \\
\hline Equal & $7(n=36)$ & $3(\mathrm{n}=36)$ \\
\hline Unequal & $3(\mathrm{n}=36)$ & $3(\mathrm{n}=34)$ \\
\hline
\end{tabular}

Additive

\begin{tabular}{|c|c|c|}
\hline & Nonflexible & Flexible \\
\hline Equal & $0(\mathrm{n}=10)$ & $0(\mathrm{n}=7)$ \\
\hline Unequal & $0(\mathrm{n}=7)$ & $0(\mathrm{n}=9)$ \\
\hline
\end{tabular}

Low

\begin{tabular}{|c|c|c|}
\hline & Nonflexible & Flexible \\
\hline Equal & $1(\mathrm{n}=8)$ & $0(\mathrm{n}=11)$ \\
\hline Unequal & $1(\mathrm{n}=10)$ & $1(\mathrm{n}=8)$ \\
\hline
\end{tabular}

Medium

\begin{tabular}{|c|c|c|}
\hline & Nonflexible & Flexible \\
\hline Equal & $1(n=9)$ & $2(n=11)$ \\
\hline Unequal & $4(n=9)$ & $2(n=8)$ \\
\hline
\end{tabular}

High

\begin{tabular}{|c|c|c|}
\hline & Nonflexible & Flexible \\
\hline Equal & $2(n=9)$ & $1(n=7)$ \\
\hline Unequal & $1(n=8)$ & $0(n=9)$ \\
\hline
\end{tabular}


Table 20: Poisson Model Parameter Estimates for

Bankruptcies: Superadditive Environments

\begin{tabular}{|c|c|c|}
\hline Variable & Estimates & t-value \\
\hline Intercept & -1.39 & -2.25 \\
\hline Flexible & -0.31 & -1.2 \\
\hline Unequal & 0.24 & 0.94 \\
\hline Level of Synergy (M, H) & $-0.31,0.76$ & $1.6,0.11$ \\
\hline CE exists & 0.17 & 0.66 \\
\hline Overlap & -.24 & -1.15 \\
\hline
\end{tabular}


Table 21: Average Withdrawals Per Auction

All Auctions

\begin{tabular}{|c|c|c|}
\hline & Nonflexible & Flexible \\
\hline Equal & $25(\mathrm{n}=36)$ & $19(\mathrm{n}=36)$ \\
\hline Unequal & $14(\mathrm{n}=34)$ & $17(\mathrm{n}=34)$ \\
\hline
\end{tabular}

Additive

\begin{tabular}{|c|c|c|}
\hline & Nonflexible & Flexible \\
\hline Equal & $3(\mathrm{n}=10)$ & $1(\mathrm{n}=7)$ \\
\hline Unequal & $0(\mathrm{n}=7)$ & $0(\mathrm{n}=9)$ \\
\hline
\end{tabular}

\section{Low}

\begin{tabular}{|c|c|c|}
\hline & Nonflexible & Flexible \\
\hline Equal & $9(\mathrm{n}=8)$ & $5(\mathrm{n}=11)$ \\
\hline Unequal & $2(\mathrm{n}=8)$ & $7(\mathrm{n}=8)$ \\
\hline
\end{tabular}

\section{Medium}

\begin{tabular}{|c|c|c|}
\hline & Nonflexible & Flexible \\
\hline Equal & $4(\mathrm{n}=9)$ & $9(\mathrm{n}=11)$ \\
\hline Unequal & $6(\mathrm{n}=9)$ & $6(\mathrm{n}=8)$ \\
\hline
\end{tabular}

High

\begin{tabular}{|c|c|c|}
\hline & Nonflexible & Flexible \\
\hline Equal & $9(\mathrm{n}=9)$ & $4(\mathrm{n}=7)$ \\
\hline Unequal & $6(\mathrm{n}=8)$ & $4(\mathrm{n}=9)$ \\
\hline
\end{tabular}


Table 22: Poisson Model Parameter Estimates for Withdrawals: Superadditive Environment

\begin{tabular}{|c|c|c|}
\hline Variable & Estimates & t-value \\
\hline Intercept & -0.24 & -0.74 \\
\hline Flexible & -0.03 & -0.29 \\
\hline Unequal & 0.11 & 0.97 \\
\hline Environment (M, H) & $-0.04,-0.05$ & $0.29,0.55$ \\
\hline CE exists & 0.17 & -0.48 \\
\hline Overlap & -0.05 & 1.39 \\
\hline
\end{tabular}


Table 23: Unsold Withdrawals Per Auction

\begin{tabular}{|c|c|c|}
\hline Treatments & Nonflexible & Flexible \\
\hline Equal & 9 out of 25 & 12 out of 22 \\
\hline Unequal & 6 out of 14 & 9 out of 21 \\
\hline
\end{tabular}


Table 24: Mean (Median) Efficiency by Auction: Additive Environment

\begin{tabular}{|c|c|}
\hline & \% Efficiency \\
\hline SMA & $96.6(100)$ \\
\hline CMA & $99.2(100)$ \\
\hline
\end{tabular}


Table 25: Count of 100\% Efficiency Outcomes by Auction Additive Environment ( $n=$ number of total experiments)

\begin{tabular}{|c|c|}
\hline & $\mathbf{1 0 0 \%}$ Efficient Outcomes \\
\hline SMA & $11(\mathrm{n}=17)$ \\
\hline CMA & $10(\mathrm{n}=11)$ \\
\hline
\end{tabular}


Table 26: Mean (Median) Efficiency by Treatment: Superadditive Environments

\begin{tabular}{|c|c|c|c|}
\hline & Low & Medium & High \\
\hline SMA & $80.6(82.5)$ & $79.0(92.0)$ & $77.1(84.0)$ \\
\hline CMA & $96.2(99.0)$ & $96.1(100)$ & $98.0(100)$ \\
\hline
\end{tabular}


Table 27: Normalized Mean (Median) Revenue: Additive Environment

\begin{tabular}{|c|c|}
\hline Treatment & Revenue \\
\hline SMA & $1.36(1.38)$ \\
\hline CMA & $1.28(1.26)$ \\
\hline
\end{tabular}


Table 28: Normalized Mean (Median) Revenue: Superadditive Environment

\begin{tabular}{|c|c|c|c|}
\hline Treatment & Low & Medium & High \\
\hline SMA & $.85(.83)$ & $.95(.92)$ & $1.05(1.00)$ \\
\hline CMA & $.77(.81)$ & $.89(.91)$ & $.90(.91)$ \\
\hline
\end{tabular}


Table 29: Average Rounds per Auction Type

\begin{tabular}{|c|c|}
\hline Treatment & Rounds \\
\hline SMA & $8.9(9)$ \\
\hline CMA & $29.3(30)$ \\
\hline
\end{tabular}


Table 30: Efficiency Results for Boundary Cases

\begin{tabular}{|l|l|c|l|l|l|}
\hline Case & Auction & \# of Auctions & Gain & Own & Efficiencies \% \\
\hline 1 & CMA & 3 & 1.23 & Yes & $78,79,78$ \\
\hline & SMA & 1 & 1.23 & Yes & 59 \\
\hline & CMA & 2 & 1.23 & No & 97,79 \\
\hline & SMA & 1 & 1.23 & No & 63 \\
\hline & CMA & 2 & 1.43 & Yes & 100,100 \\
\hline & SMA & 1 & 1.43 & Yes & 70 \\
\hline 2a & CMA & 7 & 1.25 & Yes & $99,99,99,95,94,95,95$ \\
\hline & SMA & 4 & 1.25 & Yes & $100,99,95,95$ \\
\hline $2 b$ & CMA & 3 & 1.07 & Yes & $91,94,94$ \\
\hline & SMA & 1 & 1.07 & Yes & 100 \\
\hline & CMA & 2 & 1.07 & No & 95,95 \\
\hline & SMA & 1 & 1.07 & No & 100 \\
\hline & CMA & 2 & 1.25 & Yes & 100,91 \\
\hline & SMA & 1 & 1.25 & Yes & 100 \\
\hline
\end{tabular}


Figure 1: Location Synergies

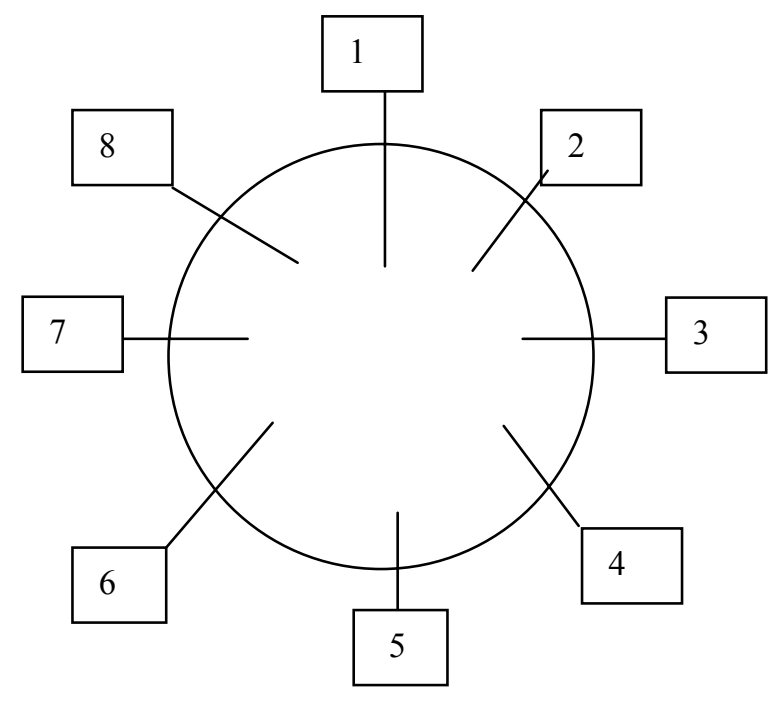


Figure 2: Inefficient License Allocation and Points

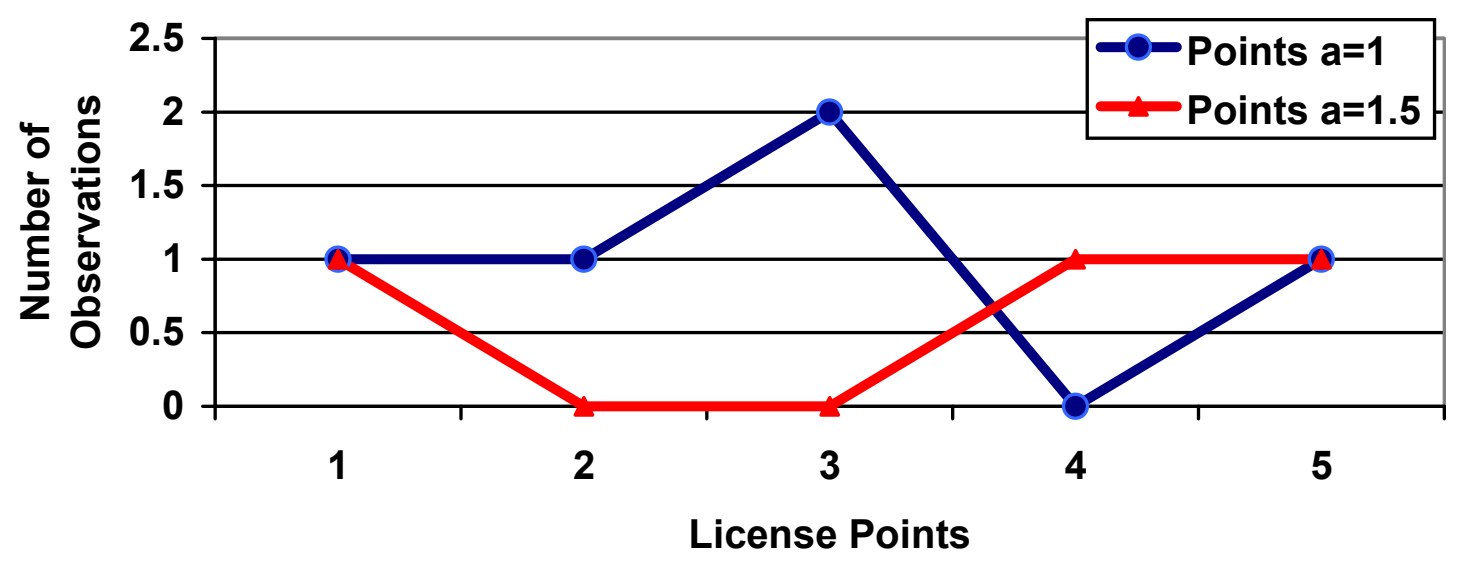


Figure 3. Relative Prices: Additive Values

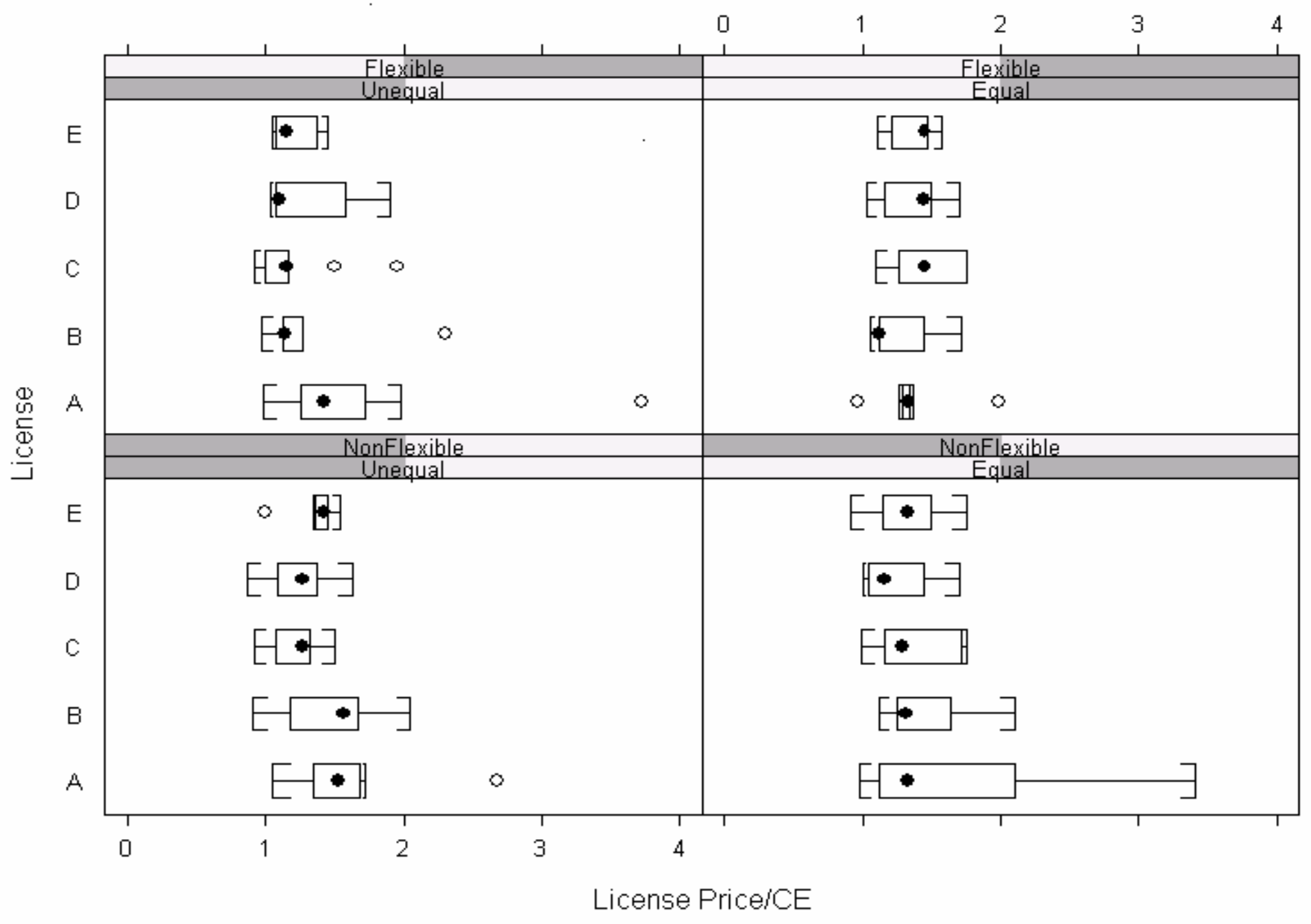


Figure 4. Relative Prices: Superadditive Values

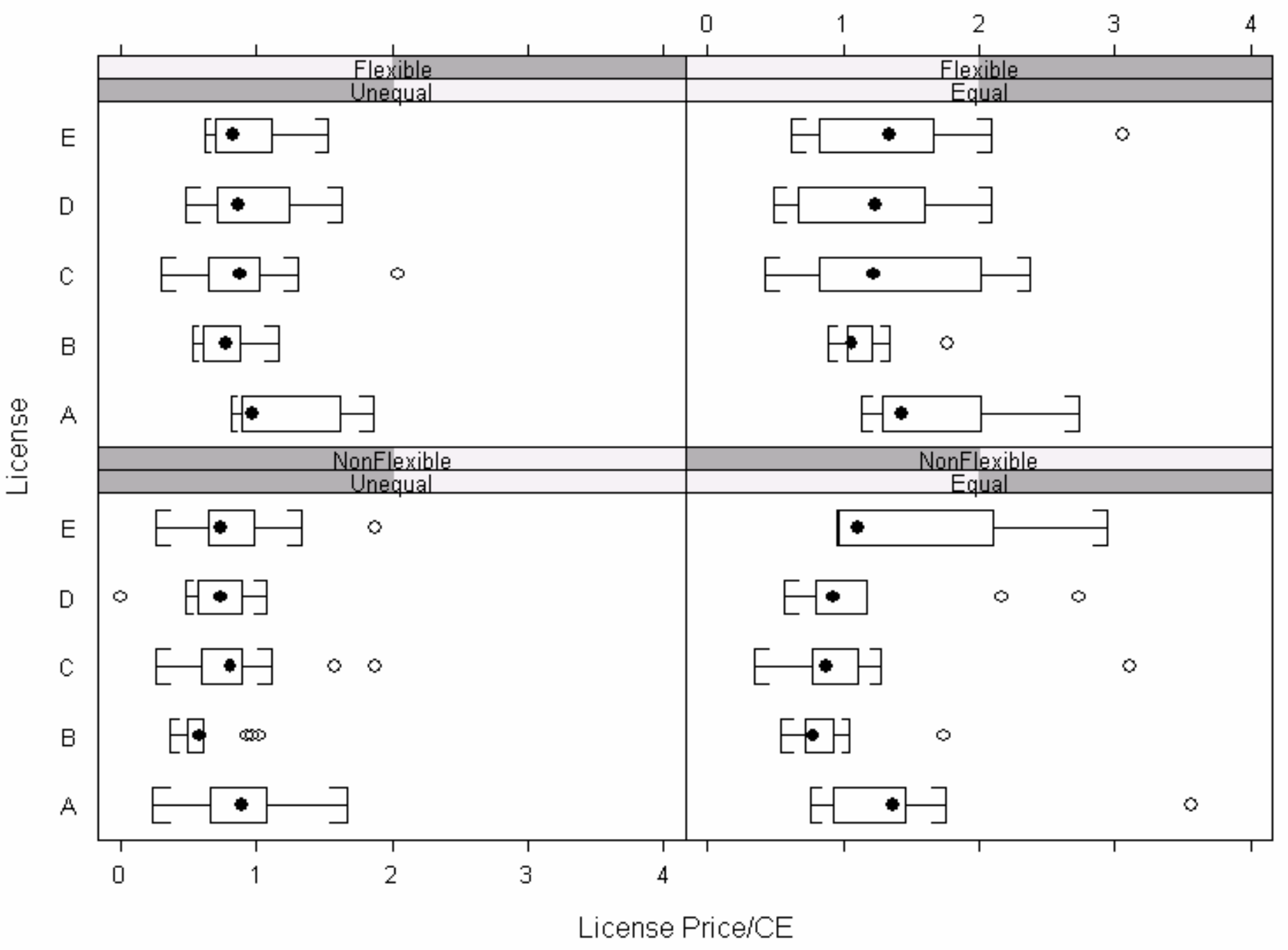




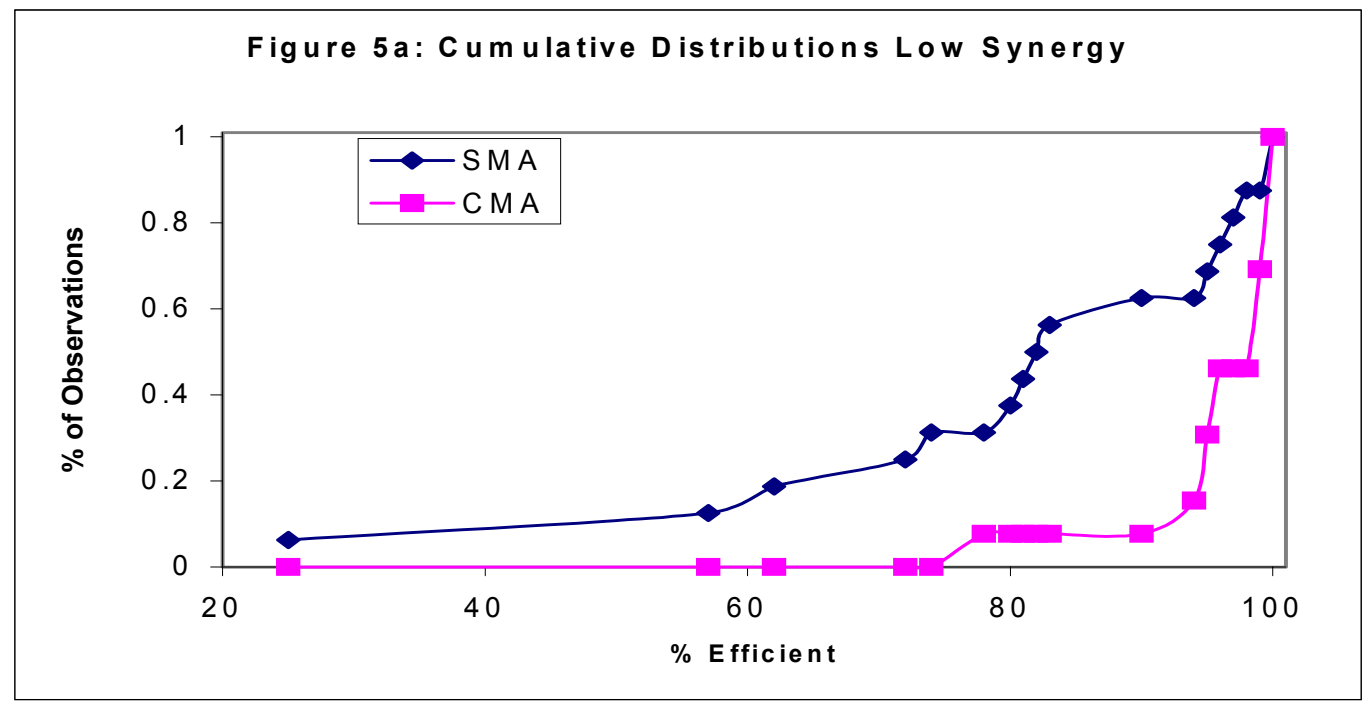




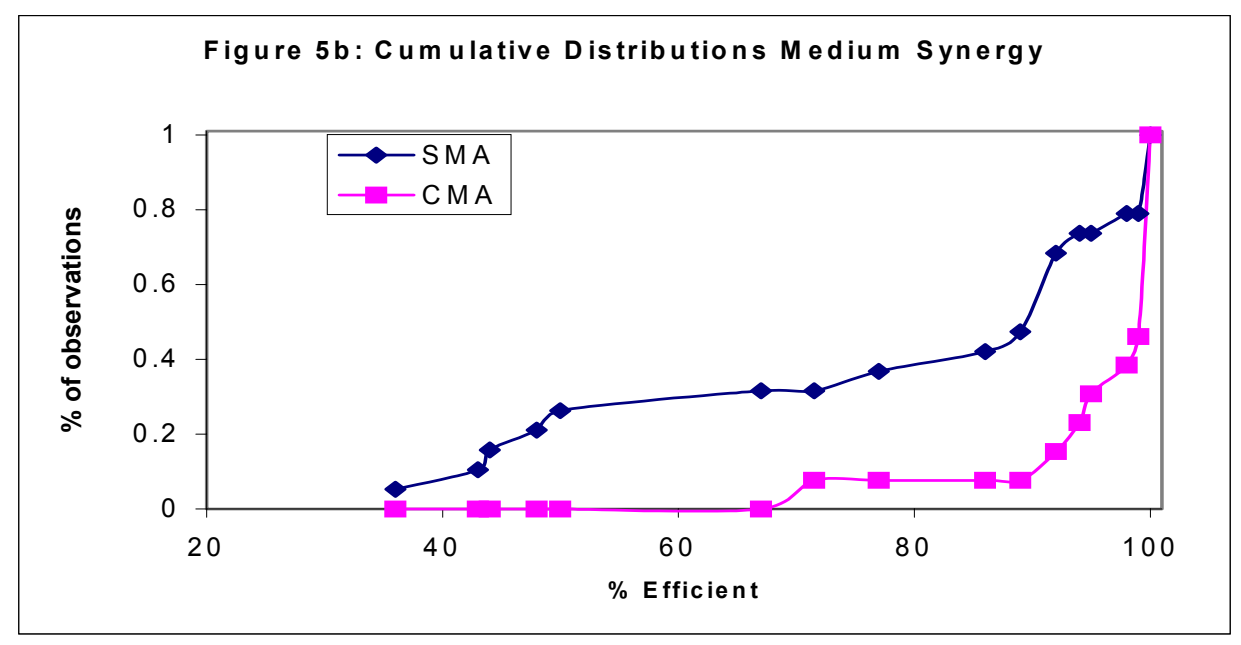




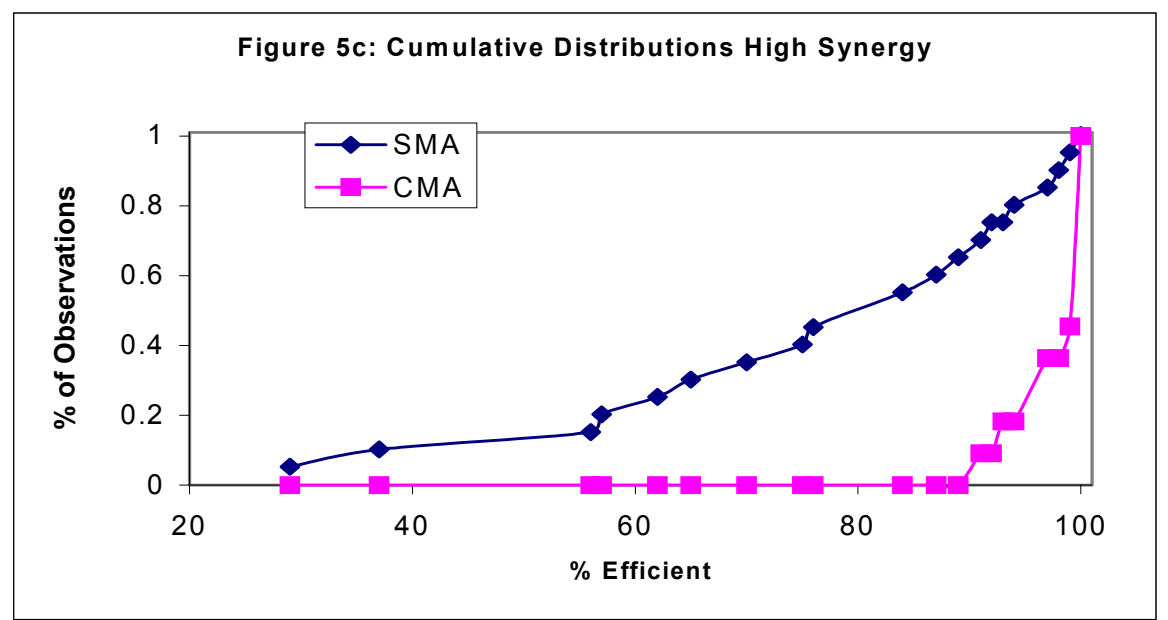


Figure 6: Gain and Own Effect Case1

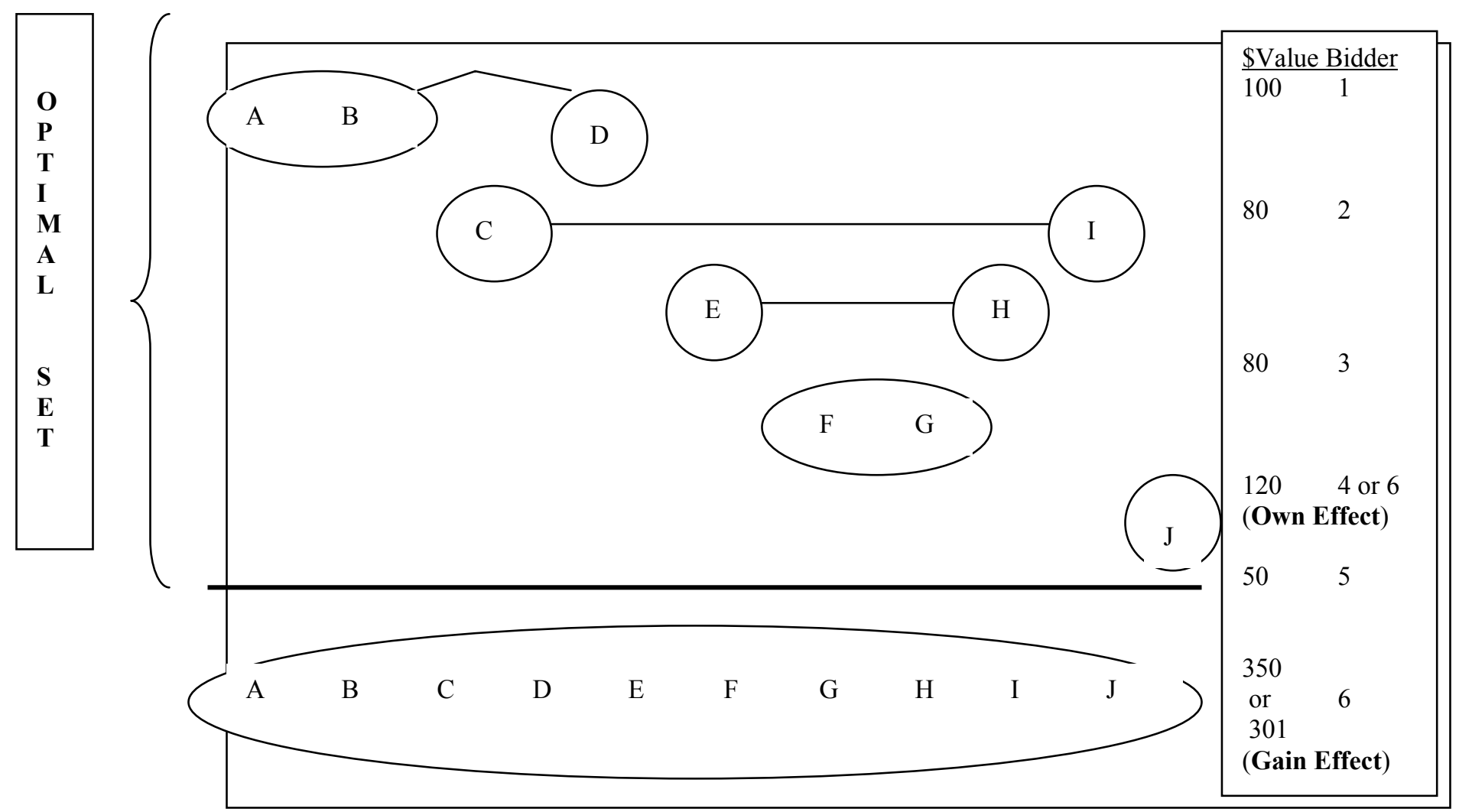


Figure 7: Gain and Own Effect Case2

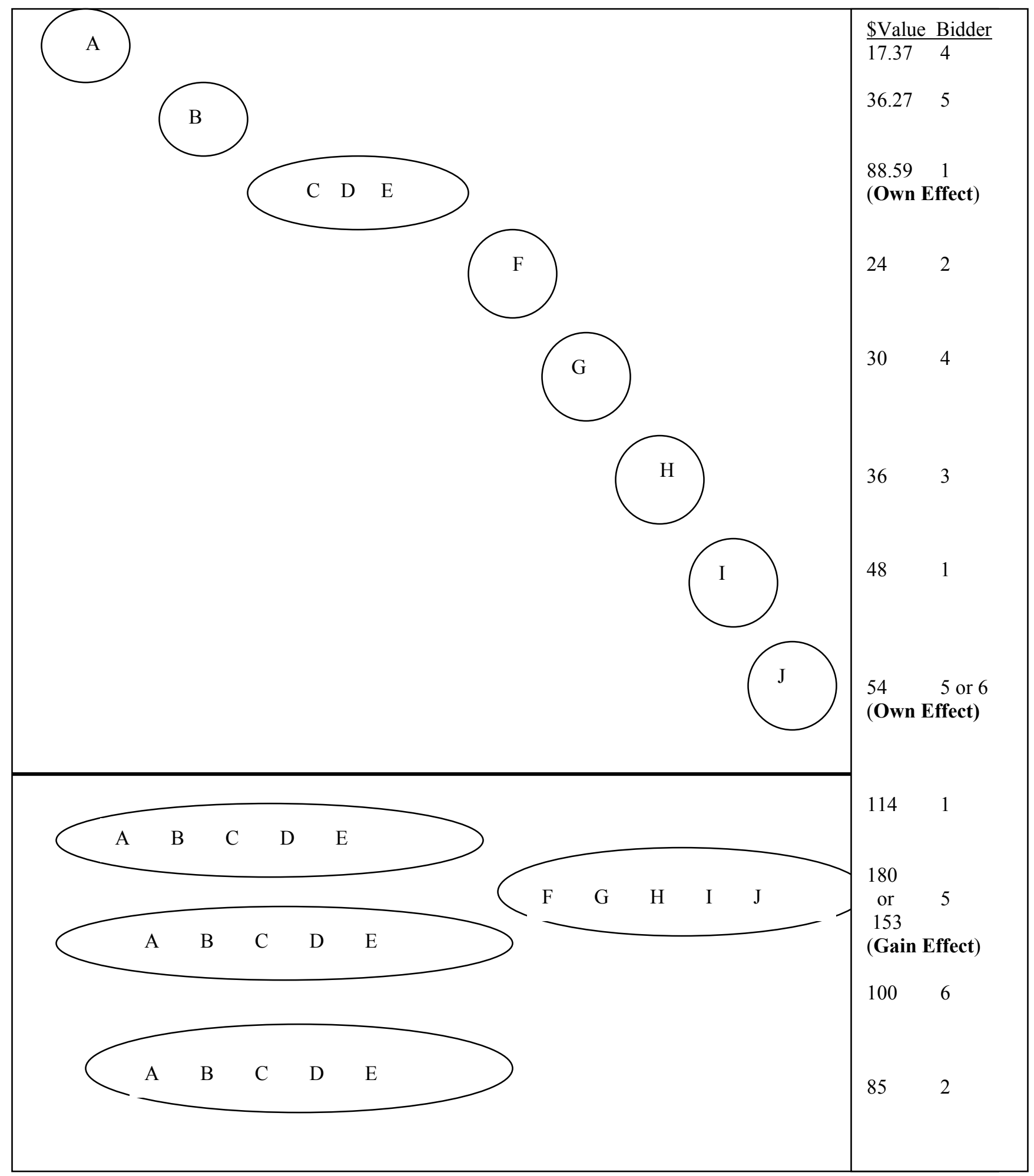

